\title{
Relativistic Jets from AGN Viewed at Highest Angular Resolution
}

\author{
Kazuhiro Hada \\ Mizusawa VLBI Observatory, National Astronomical Observatory of Japan, 2-12 Hoshigaoka, Mizusawa, \\ Oshu, Iwate 023-0861, Japan; kazuhiro.hada@nao.ac.jp; Tel.: +81-197-22-7129
}

Received: 31 October 2019; Accepted: 2 December 2019; Published: 18 December 2019

\begin{abstract}
Accreting supermassive black holes in active galactic nuclei (AGN) produce powerful relativistic jets that shine from radio to $\mathrm{GeV} / \mathrm{TeV} \gamma$-rays. Over the past decade, AGN jets have extensively been studied in various energy bands and our knowledge about the broadband emission and rapid flares are now significantly updated. Meanwhile, the progress of magnetohydrodynamic simulations with a rotating black hole have greatly improved our theoretical understanding of powerful jet production. Nevertheless, it is still challenging to observationally resolve such flaring sites or jet formation regions since the relevant spatial scales are tiny. Observations with very long baseline interferometry (VLBI) are currently the only way to directly access such compact scales. Here we overview some recent progress of VLBI studies of AGN jets. As represented by the successful black hole shadow imaging with the Event Horizon Telescope, the recent rapid expansion of VLBI capability is remarkable. The last decade has also seen a variety of advances thanks to the advent of RadioAstron, GMVA, new VLBI facilities in East Asia as well as to the continued upgrade of VLBA. These instruments have resolved the innermost regions of relativistic jets for a number of objects covering a variety of jetted AGN classes (radio galaxies, blazars, and narrow-line Seyfert 1 galaxies), and the accumulated results start to establish some concrete (and likely universal) picture on the collimation, acceleration, recollimation shocks, magnetic field topology, and the connection to high-energy flares in the innermost part of AGN jets.
\end{abstract}

Keywords: active galaxies; supermassive black holes; relativistic jets; high-resolution radio observations

\section{Introduction}

A small fraction of galaxies release an enormous amount of energy from a tiny volume of the central regions that outshine the whole light of the host galaxies. They are called active galactic nuclei (AGN) and now are widely believed to be powered by the accretion onto the supermassive black holes (SMBH) [1]. Approximately 10 percent of AGN are radio-loud and exhibit powerful relativistic jets [2], a collimated beam of nonthermal plasma that often propagates beyond the host galaxy. Relativistic jets in AGN have attracted astronomers over the whole past century since the first discovery of such phenomena in the elliptical galaxy M87 [3]. Understanding the formation processes of AGN jets is one of the primary goals in high-energy astrophysics. Moreover, AGN jets transport a significant fraction of $\mathrm{BH}$ accretion energy back into their host galaxies and intergalactic space, so studies of AGN jets are also important for understanding the formation and cosmological evolution of galaxies.

The study of AGN jets is challenging for a number of reasons. First, the physics of jet dynamics is extremely complicated. As a jet consists of relativistic magnetized fluids launched near a SMBH and the inner part of magnetized accretion flows, its generation and propagation requires a full treatment of general relativistic magnetohydrodynamics (GRMHD) equations, which has just become possible in recent years thanks to the rapid progress of numerical simulations (see, e.g., in $[4,5]$ ). Besides, the processes of particle acceleration, energy dissipation (e.g., shocks, magnetic reconnection, 
turbulence) and their location in the jet are still not well understood, leaving a variety of models to reproduce the observed broadband emission (see, e.g., in [6-10]). Second, important jet physical processes as described above are generally associated with very compact spatial scales. According to the current theoretical paradigm of AGN jet production, a jet is initially produced by strong magnetic fields ( $B$-fields) that are amplified by a spinning black hole and the inner part of accretion flows $[11,12]$. The flow is initially slow in a magnetically dominated state, and the subsequent acceleration is realized by gradually converting the magnetic energy into kinetic energy. Consequently, the theories suggest that an acceleration and collimation zone (ACZ) is formed over distances $\sim 10-10^{5}$ Schwarzschild radii $\left(R_{\mathrm{S}}\right)$ from the $\mathrm{BH}$ (e.g., [13]). These scales correspond to milliarcsecond (mas) or microarcsecond ( $\mu$ as) scales for nearby $(z<0.1)$ AGN jets with $10^{8-9}$ SMBH masses. Also, rapid variability and flares observed in X-ray/ $\gamma$-ray bands often suggest the size of high-energy emitting site to be sub-parsec (pc) scales or even less. Such tiny scales are hard to directly resolve with optical/X-ray/ $\gamma$-ray instruments.

To directly access such compact scales, very long baseline interferometer (VLBI) observations at radio wavelengths are necessary. Early VLBI experiments with ad hoc arrays from the 1970s to 1980s revealed the pc-scale structures in a few brightest radio sources (see, e.g., in [14-17]) with the discovery of core-jet morphology and superluminal motions that are now routinely seen in many jets. The advent of the Very Long Baseline Array (VLBA) ${ }^{1}$ in the 1990s greatly enhanced high-resolution studies of AGN jets thanks to its exclusive use for VLBI. AGN jets are now widely studied also with various arrays such as the European VLBI Network $(E V N)^{2}$, the Long Baseline Array (LBA $)^{3}$ and the East Asian VLBI Network (EAVN) ${ }^{4}$ [18]. These networks were occasionally connected to space-VLBI satellites such as VSOP (1997-2005) [19] and RadioAstron (2012-2019) [20], further enhancing angular resolution. Although the above facilities are mainly operated between 1 and $43 \mathrm{GHz}, \mathrm{VLBI}$ is becoming more common also at higher frequencies $86 / 129 \mathrm{GHz}$, as represented by the Global Millimeter VLBI Array $(\mathrm{GMVA})^{5}$ and the Korean VLBI Network $(\mathrm{KVN})^{6}$. Ultimately, at the highest frequencies, the rapid expansion of the Event Horizon Telescope $(\mathrm{EHT})^{7}$, a global $230 \mathrm{GHz}$ (and $350 \mathrm{GHz}$ ) VLBI array, is touching the horizon-scale structures of nearby AGN thanks to its unprecedented high angular resolution ( $\sim 20 \mu \mathrm{as})$ and reduced optical depth towards the radio core, opening a new frontier of observational studies of AGN/SMBH.

In short, the merits of VLBI for AGN-jet studies can be summarized as follows:

- High-resolution imaging — this allows us to determine the shapes and transverse structures of the innermost jet regions robustly.

- Structural evolution monitoring-this allows us to investigate the kinematics and velocity structures of the innermost jet regions via multi-epoch observations.

- Spatially-resolved polarimetry - this allows us to probe the detailed configuration the $B$-fields associated with jets through measurements of electric vector polarization angles (EVPA) or Faraday rotation measure (RM).

Therefore, these features of VLBI are all closely relevant to the key questions on AGN jets, such as the collimation, acceleration, and role of $B$-fields. Moreover, by coordinating VLBI sessions with other multi-wavelength (MWL) facilities, we can set tight constraints on the location of high-energy flares by cross-correlating the MWL data and examining possible ejections of new components.

In this review, we overview the recent progress of observational studies of AGN jets with a particular focus on VLBI. For an extensive review covering observational results on the other

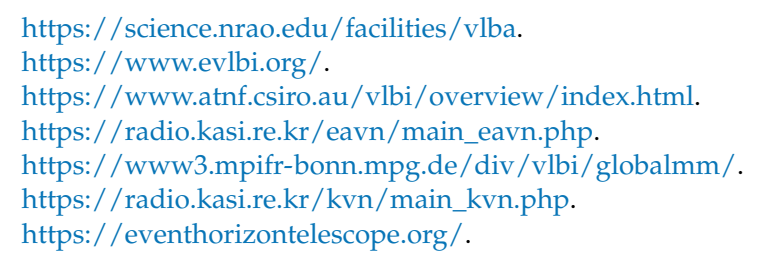


wave bands and theoretical efforts in understanding relativistic jets, please see Blandford et al. [21]. The following three sections are divided by three different classes of AGN: (1) radio galaxies, (2) blazars, and (3) narrow-line Seyfert 1 (NLSy1) galaxies. These three classes of AGN are now firmly established as the ones with powerful relativistic jets thanks to the recent accumulated data from the Fermi high-energy $\gamma$-ray satellite [22]. In Section 2, we start with radio galaxies, for which VLBI studies on individual objects have probably most advanced in recent years. In Section 3, we focus on blazars for which continued efforts on VLBI monitoring have been made over the last 2-3 decades. In Section 3, we briefly overview radio-loud NLSy1s, which have recently been identified as a new class of jetted AGN. Finally, in Section 4, we provide a summary with some future prospects.

\section{Radio Galaxies}

Radio galaxies are a major subclass of radio-loud AGN, where the jet axis is misaligned from our line-of-sight $\left(i \geq 10^{\circ}\right)$. Due to their milder Doppler boosting effect than that of blazars, radio galaxies often show the morphology of two-sided jets plus core at various scales. Radio galaxies are further divided into two types, i.e., Fanaroff \& Riley (FR) I and II [23]. The former type sources have lower luminosity $\left(L_{178 \mathrm{MHz}} \leq 2 \times 10^{25} \mathrm{~W} \mathrm{~Hz}^{-1}\right)$ radio jets whose intensity fall away from the nucleus, whereas the latter have higher-power jets leading to hot spots at the outermost edges of the radio lobes. Despite the misaligned nature, now more than 40 radio galaxies are detected in $\mathrm{GeV} \gamma$-rays with Fermi [24], and a few of them (e.g., 3C 84) show quite variable $\gamma$-ray activities reminiscent of blazars. Since well-known radio galaxies are located at low redshifts compared to typical blazars and also the location of the central engine can be tightly constrained as the root of a twin jet, radio galaxies are suitable targets to investigate the jet formation and collimation scales near SMBH. Here, we particularly highlight two nearby radio galaxies M87 and 3C 84, which are among the most intensively studied objects by VLBI in the last decade, and thus unprecedented details on the innermost jet structures are currently known.

\section{1. $M 87$}

M87 is a FR-I type radio galaxy at the center of the Virgo cluster. Due to its proximity $(D=16.8 \mathrm{Mpc}$ [25]), the kpc-scale jet is well resolved at radio, optical and X-rays (see, e.g., in [26-29]). Past optical/IR spectroscopic studies of the nuclear gas and stellar dynamics suggest a SMBH of $M_{\mathrm{BH}} \sim(3.2-6.6) \times 10^{9} M_{\odot}[30-32]$. The combination of the proximity and the large $M_{\mathrm{BH}}$ yields a linear resolution $1 \mathrm{mas}=0.08 \mathrm{pc}=128-265 R_{\mathrm{s}}$. Astrometric core-shift measurements indicate the $43 \mathrm{GHz}$ core to be located within a few tens of $R_{\mathrm{S}}$ from the $\mathrm{BH}$ [33], and early EHT $230 \mathrm{GHz}$ experiments detected a compact radio core with a size of $\sim 40 \mu$ as that is consistent with a horizon-scale structure [34,35]. Therefore M87 has long been considered as a privileged target for imaging a BH shadow [36] and launching/formation scales of a relativistic jet with VLBI.

\subsubsection{Black Hole Shadow}

An epoch-making result has recently been released from the EHT Collaboration [37-42]. By assembling an Earth-sized $230 \mathrm{GHz}$ VLBI array with eight stations, including ALMA, the EHT has detected the shadow of the central SMBH of M87 (Figure 1 bottom). Some of the main features of the M87 EHT images are summarized as follows. (1) The images show a bright circular ring with a diameter of $42 \pm 3 \mu$ as with a central dark area, where the brightness ratio is $\geq 10: 1$. The ring-like structure is in agreement with a gravitationally-lensed photon ring expected by the general relativity around a Kerr spacetime. (2) The ring diameter constrains the mass of the central $\mathrm{BH}$ to be $(6.5 \pm 0.7) \times 10^{9} \mathrm{M}_{\odot}$, which is in agreement with the value estimated from the previous stellar dynamics measurements [32]. (3) The peak brightness temperature is $T_{\mathrm{B}} \sim 6 \times 10^{9} \mathrm{~K}$, which is consistent with the radio emission to have synchrotron origins. (4) The ring shows an asymmetry in brightness where the southern part is brighter than the northern part, which can be explained by relativistic Doppler boosting (deboosting) of matter rotating around the $\mathrm{BH}$. As the observed emission originates within a few gravitational radii 
from the $\mathrm{BH}$, the north-south asymmetry can be controlled by the $\mathrm{BH}$ spin and thus suggests the sense of $\mathrm{BH}$ spin to be in the clockwise direction when viewed from the Earth. Overall, these features provide compelling evidence for the presence of the $\mathrm{SMBH}$ as the central engine of AGN. Nevertheless, the first M87 EHT images show no clear signature of extended emission around the ring, despite the remarkable jet visible at low frequencies. It is likely that the first EHT images are still highly dynamic range-limited with the absence of short baselines that are sensitive to the extended emission. Future EHT observations with enhanced sensitivity and image dynamic range (e.g., using more stations) would be the key to connect the $\mathrm{BH}$ shadow to jet launching unambiguously.

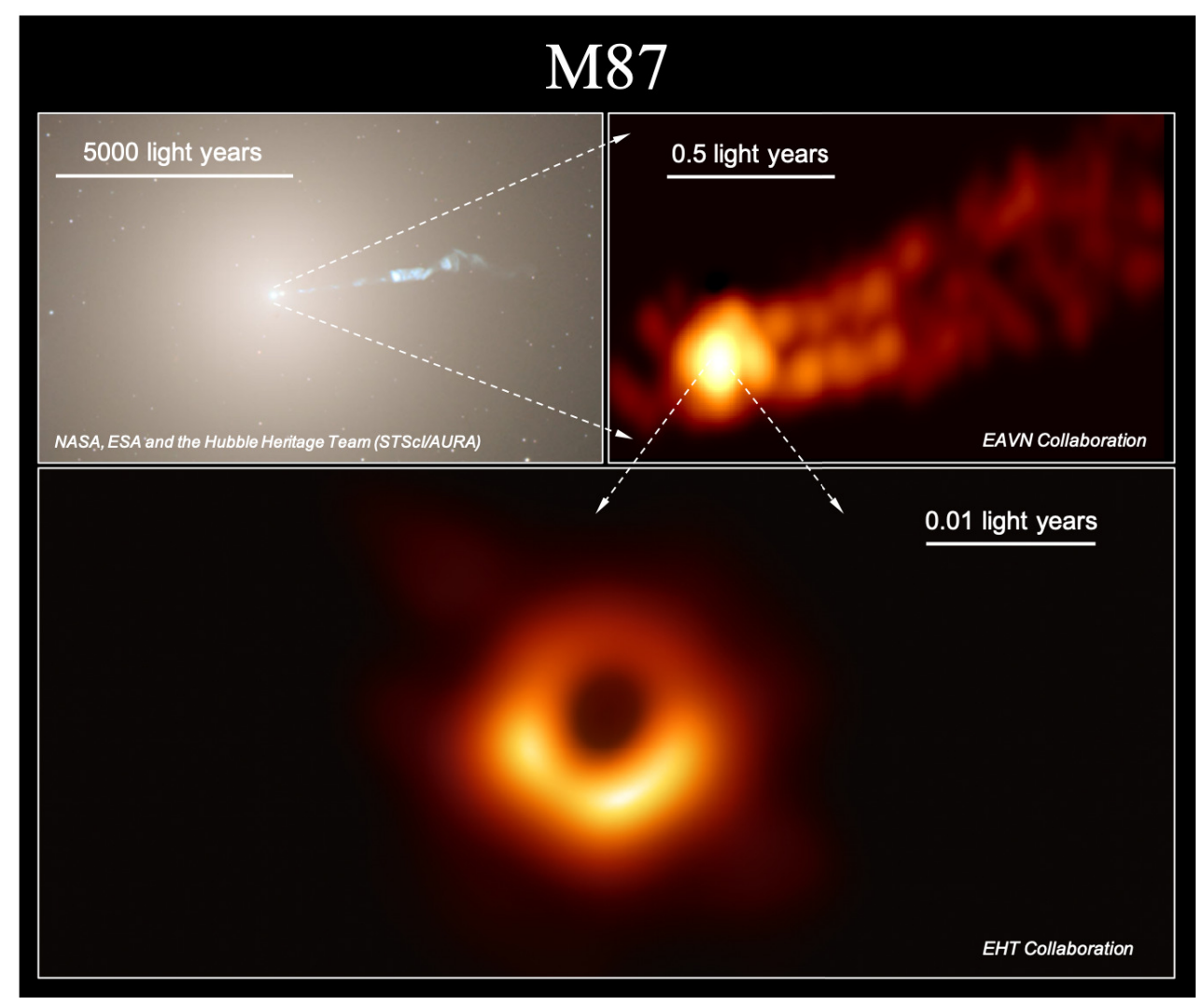

Figure 1. M87 images from galactic to event horizon scales. (Top left) HST image of M87 (credit: NASA, ESA and the Hubble Heritage Team (STScI/AURA)). (Top right) EAVN 43 GHz image of M87 (credit: EAVN Collaboration). (Bottom) EHT $230 \mathrm{GHz}$ image of M87 (credit: EHT Collaboration).

\subsubsection{Jet Base, Collimation, and Transverse Structures}

The M87 jet beyond the horizon scales has intensively been investigated over the last years with VLBI at $86 \mathrm{GHz}$ and lower frequencies (Figure 1 top right). Recent high-sensitivity array (HSA) or GMVA $86 \mathrm{GHz}$ images of M87 have resolved a wide opening angle jet base within $\leq 10-100 R_{\mathrm{S}}$ scales with a clear limb-brightening structure $[43,44]$, which is consistent with magnetically-driven jet simulations/models with a spinning $\mathrm{BH}$ [45-48]. At similar scales VLBA polarimetric images at $43 \mathrm{GHz}$ indicates the presence of hellically-wrapped $B$-fields [49], which is also suggestive of magnetic scenarios. Beyond $100 R_{\mathrm{s}}$ from $\mathrm{BH}$, multi-band VLBI images at $1-43 \mathrm{GHz}$ revealed a parabolic shape of the M87 jet all the way up to $\sim 10^{6} R_{\mathrm{S}}$ where the bright knot HST-1 appears [50-52], whereas beyond HST-1, the jet becomes conical [26,50] (Figure 2 left). Notably, the geometrical transition coincides with the Bondi radius of SMBH [53,54], suggesting that the external medium bounded by the SMBH gravity plays a key role in supporting the jet collimation [50] (see also Section 2.1.4).

Although the limb-brightened envelope is now routinely seen, some of the recent high quality M87 VLBI images at $\leq 15 \mathrm{GHz}$ have detected another streamline in the middle of the jet $[55,56]$. Such a triple-ridge structure is suggestive of a "spine sheath" structure, in which the central spine 
could be associated with a BH-powered jet (e.g., [57]). Additional observational clues (e.g., spectral and velocity gradients across jet) would better understanding of the nature of the central stream.
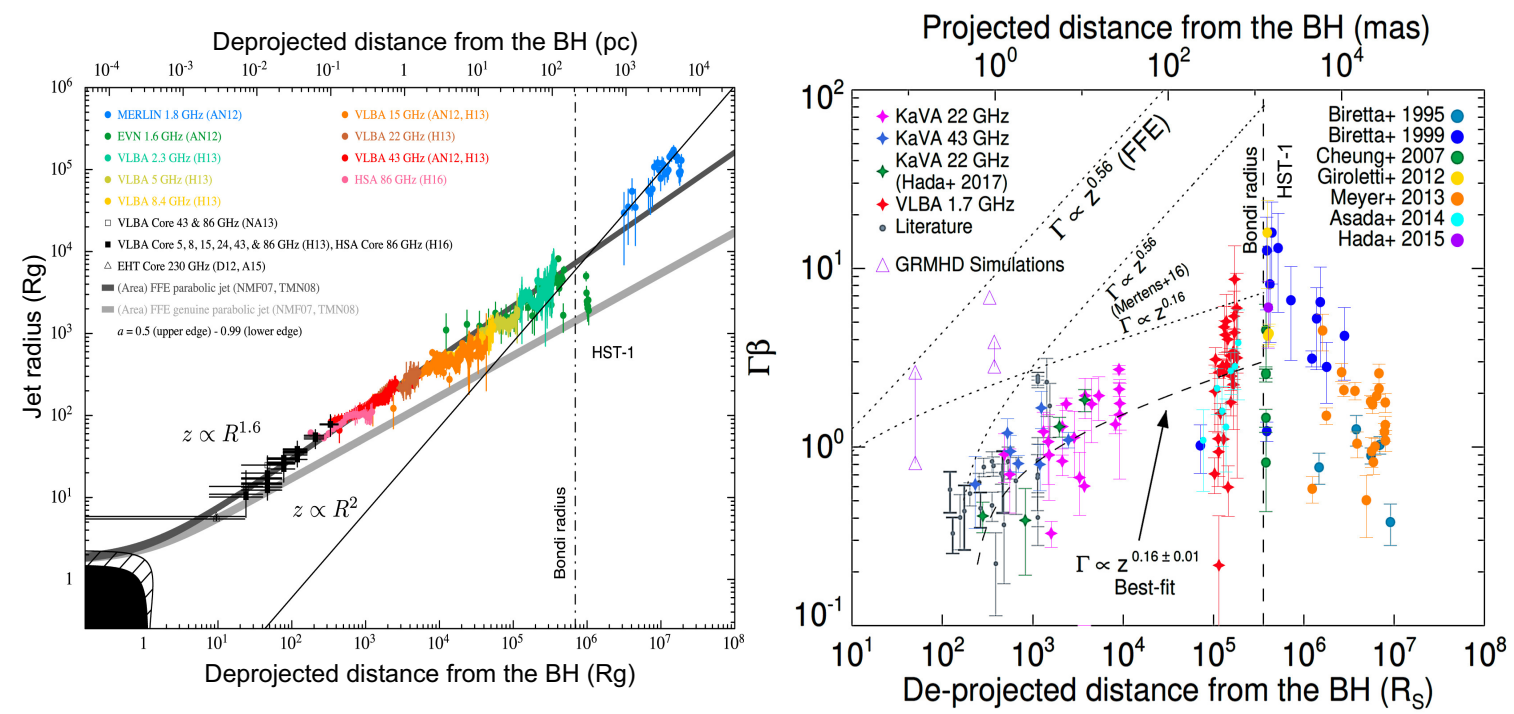

Figure 2. Collimation and acceleration of the M87 jet. (Left) Jet collimation profile of M87 (adapted from work in [47]). (Right) Jet velocity profile of M87 as a function distance (adapted from work in [58]).

\subsubsection{Jet Kinematics and Velocity Profile}

The kinematics of the M87 jet is still controversial. At kpc scales, the jet proper motion is relatively well defined [27,59], where the jet is overall superluminal and decelerating from $\sim 6 c$ at $\sim 100 \mathrm{pc}$ (HST-1) to $\sim 1 c$ at $>1000 \mathrm{pc}$. At pc-subpc scales on the other hand, past low-cadence VLBA monitoring often reported very slow or quasi-stationary speeds [60-63]. However, such low speeds would be inconsistent with the large jet-counterjet brightness ratio, implying that monthly/yearly monitoring intervals are insufficient to properly trace the inner jet motion.

In this context, several groups have started more dedicated (biweekly or less) monitoring programs of the M87 inner jet using various different facilities/frequencies (VLBA at 43 GHz [49,64,65]; $\mathrm{KaVA} / \mathrm{EAVN}$ at $22 / 43 \mathrm{GHz}[58,66]$ ) (Figure 2 right). Importantly, they consistently detected fast motions (up to $\sim 2-3 c$ ) within a few $100 R_{\mathrm{S}}$ from the jet base, which can account for the jet-counterjet ratio. They also found a trend of gradual acceleration with distance that may connect to the maximum speed at HST-1. Notably, the observed acceleration regions seem to be coincident with those where the parabolic jet collimation is seen. This implies that the collimation and acceleration are closely linked with each other for a wide range of distances from $\mathrm{BH}$, as expected by the magnetic collimation and acceleration scenarios $[13,47]$.

Nevertheless, these studies also claim the presence of multiple velocity components, even at the same distances from $\mathrm{BH}$, suggesting that the true jet velocity fields are more complicated. This may indicate that one speed is associated with a bulk flow while another one traces a pattern or instability filament [65]. Alternatively, the jet contains velocity stratification where different jet speeds represent different layers in the jet [58]. Further accumulation of velocity measurements/samples would be required to discriminate between these scenarios.

\subsubsection{Accretion Flows and Winds}

The nucleus of M87 is known to be highly underluminous $\left(L_{\mathrm{bol}} \sim 10^{42} \mathrm{erg} \mathrm{s}^{-1} \sim 10^{-6} L_{\text {Edd }}\right.$ [67] $)$ compared to the luminosity expected from the accretion rate $(\dot{M})$ determined at the Bondi radius $\left(\dot{M}_{\mathrm{B}} \sim 0.2 \mathrm{M}_{\odot} \mathrm{yr}^{-1}[53,54]\right)$. This indicates that the accretion state of the M87 BH is highly radiatively inefficient and/or $\dot{M}$ near the horizon is much lower than $\dot{M}_{\mathrm{B}}$. Indeed, RM measurements of the nucleus with the submillimeter array (SMA) at $230 \mathrm{GHz}$ show $\dot{M} \leq 10^{-3} \mathrm{M}_{\odot} \mathrm{yr}^{-1}$ near the $\mathrm{BH}$, at least 
2 orders of magnitude lower than $\dot{M}_{\mathrm{B}}$ [68], suggesting a substantial mass loss between the Bondi radius and $\mathrm{BH}$. However, there were no dedicated studies searching for the inferred mass loss between these two scales.

In this context, by collecting multi-frequency VLBA data, Park et al. [69] have recently examined the spatially-resolved RM structures of M87 inside the Bondi radius in great detail. They discovered a systematic decrease of RM magnitude with distance between 5000 and 200,000 $R_{\mathrm{S}}$ from the $\mathrm{BH}$ (Figure 3), the slope of which can be nicely reproduced with a thermal gas density profile $\rho \propto r^{-1}$. This density profile is significantly flatter than that of the classical advection-dominated accretion flows (ADAF) with pure inflows, and strongly indicates a mass loss with substantial winds as expected by the adiabatic inflow-outflow solution (ADIOS) [70]. Winds from radiatively inefficient accretion flows (RIAF) have long been considered theoretically, but this work provides the first compelling observational evidence confirming the prediction. Furthermore, the RM results also confirmed that the pressure profile of the winds is shallow enough to confine the M87 jet in a parabolic shape until the Bondi radius. This is also in good agreement with recent GRMHD jet simulations with RIAF-type flows [47], where winds from RIAF are indeed the major external source confining the relativistic jet.

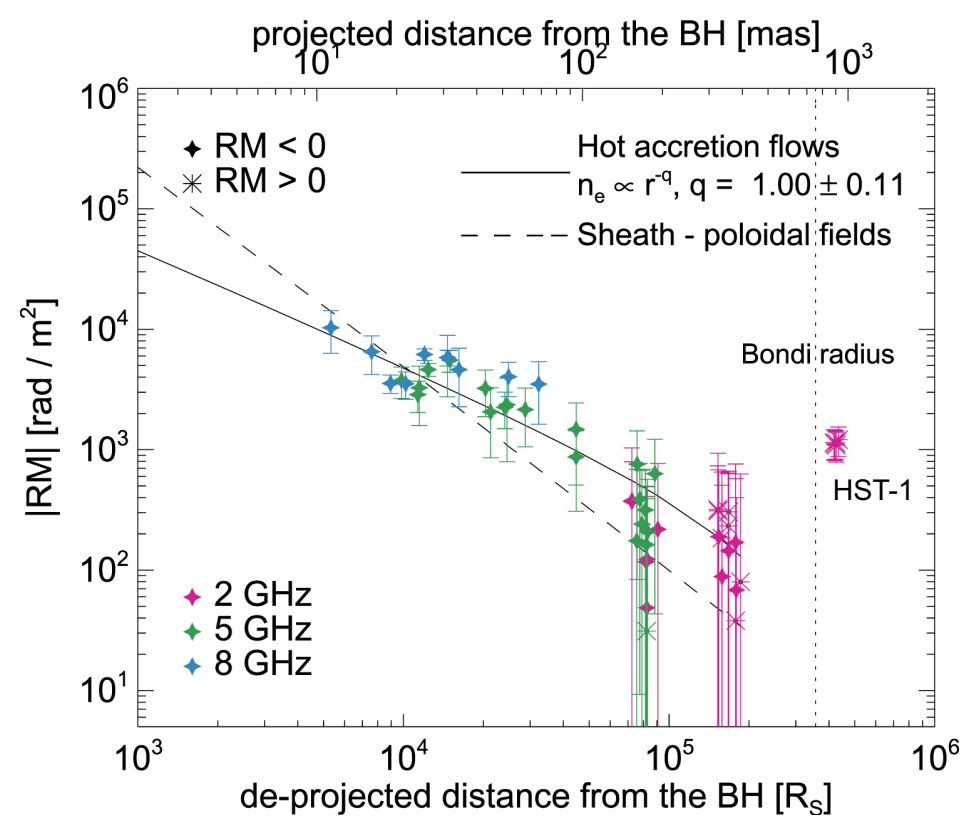

Figure 3. VLBA RM profile of the M87 jet as a function of distance from the BH (adapted from work in [69]).

\subsubsection{Connection to High-Energy Emission}

M87 is also known as a $\gamma$-ray emitter up to $\mathrm{GeV} / \mathrm{TeV}$ bands, and joint VLBI monitoring, along with $\gamma$-ray instruments, plays a key role in pinpointing the location of high-energy emission site. To date, M87 underwent three remarkable TeV flaring events in 2005, 2008, and 2010. In 2005, a TeV flare [71] was accompanied by the radio-to-X-ray outbursts from HST-1 with the emergence of superluminal components [72]. For the 2008/2010 events, in contrast, the nucleus showed enhanced emission at $43 \mathrm{GHz}$ and X-rays [73-76]. A relatively minor TeV event was also reported in 2012 [77], where the radio core again showed a remarkable flux increase while HST-1 remained quiescent [78]. This event also coincided with a $230 \mathrm{GHz}$ VLBI session on the nucleus, and interestingly it detected an enhanced $230 \mathrm{GHz}$ flux not on horizon scales but on $\sim 30-60 R_{\mathrm{s}}$ scales, suggesting that the active site may not be too close to the $\mathrm{BH}$ [35]. In summary, the previous MWL statistics tend to prefer the core to HST-1 as the origin of $\gamma$-ray production. Nevertheless, the events associated with the core all showed different types of radio-TeV correlation, leaving a variety of theoretical models still viable (see, e.g., in [79-84]) (see, also, review in [85]). 
Note that, despite its prolonged low state after the 2005 event, HST-1 still continues to attract a great deal of interest. A continuing HST-1 monitoring with EVN/VLBA indicates the persistent fast (4-6) $c$ motion with repeating new ejections from the upstream side [86,87], resembling the core of $\gamma$-ray blazars. A recent VLBA polarimetric study of M87 found a significant local increase of RM values in HST-1 (Figure 3), further supporting the recollimation shock scenario of this feature [69].

\section{2. $3 C 84$}

3C 84 is a nearby $(D=75 \mathrm{Mpc}$ ) bright radio source associated with the elliptical galaxy NGC 1275 in the Perseus cluster. The radio source is known to have multiple lobe-like morphology from $10 \mathrm{kpc}$ to pc scales [88,89], imprinting recurrent jet activities. Between 2005 and 2008, the nucleus restarted its activity with enhanced $\gamma$-ray emission [90], followed by the ejection of a new radio component "C3" from the subpc-scale core towards the southern jet [91,92]. This led to subsequent extensive MWL observations of $3 \mathrm{C} 84$ that are still ongoing. Various observations of this galaxy indicate gas-rich nuclear environments feeding the AGN [93-99]. These properties make 3C 84 a unique target for examining the connection among jet formation, $\gamma$-ray production, feeding/accretion to $\mathrm{SMBH}$, and feedback to the environments.

Recent high-resolution VLBI imaging of 3C 84 has significantly updated our knowledge about the transverse structure of the pc-subpc-scale jet. With VLBA at $43 \mathrm{GHz}$ Nagai et al. [100] revealed a clear limb-brightened morphology on the restarted jet. Moreover, RadioAstron has resolved the limb-brightened structure even closer to the jet base down to $50 \mu$ as scales (corresponding to $\sim 200 R_{\mathrm{S}}$ ) [101] (Figure 4 left). The image discovered a broad opening angle near the jet base similar to M87, suggesting that the jet formation scales near $\mathrm{BH}$ is beginning to be accessed. Intriguingly, the subsequent collimation profile is close to cylindrical, in contrast to M87. This implies the difference in the environments where the jet propagates, given the gas-rich properties known on large scales. However, the circumnuclear properties on small $(\sim p c)$ scales have not been examined yet.
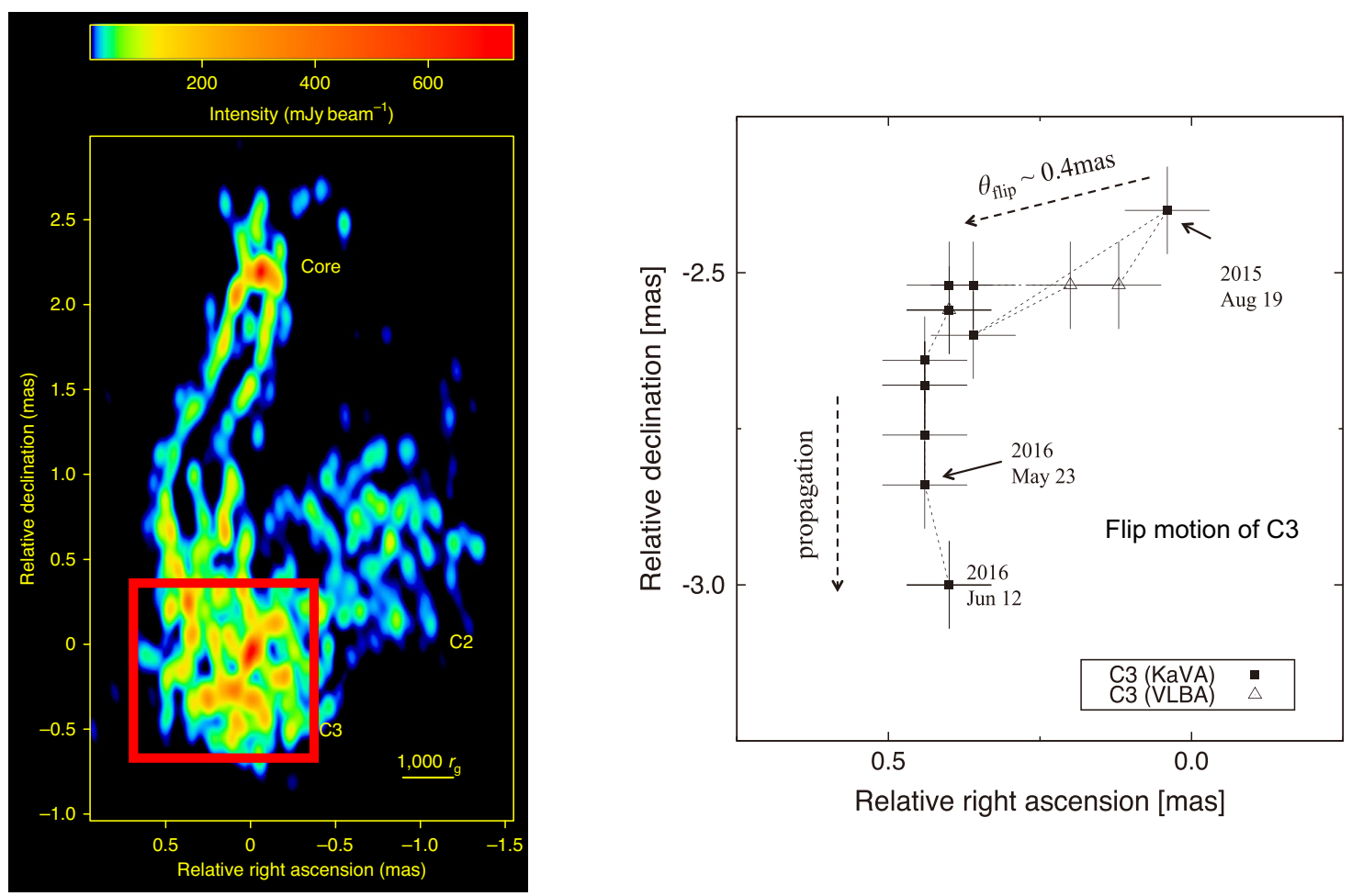

Figure 4. (Left) RadioAstron $22 \mathrm{GHz}$ image of 3C 84 observed in 2013 (adapted from work in [101]). (Right) Trajectory of C3 from August 2015 to June 2016, obtained by KaVA and VLBA at 43 GHz (adapted from work in [102]). A red rectangular area overlaid on the RadioAstron image corresponds to the plotting area of the $\mathrm{C} 3$ trajectory. 
In this context, some clues to the pc-scale circumnuclear environments have been found by monitoring the long-term trajectory of C3: the head of the restarted jet. While C3 was traveling straight towards the south after its ejection [103], during mid 2015, C3 showed a sudden change of direction, moving towards the east by 0.4 mas ( 0.14 pc in linear scale) [102] (Figure 4 right), together with enhanced radio emission in both total $[102,104]$ and polarized fluxes [105]. This peculiar behavior is hard to be explained by the intrinsic motion of the jet, whereas it would be naturally explained if C3 is interacting with an inhomogeneous dense clumpy medium [106]. The presence of inhomogeneous, dense surrounding medium at pc-subpc scales is also suggested by the recent detection (and free-free absorbed spectra) of the counter-jet at 2 mas north from the core [107].

The dense circumnuclear environments, on the other hand, could make the study of intrinsic jet properties and their connection to the $\gamma$-ray production more complicated. Therefore, high-frequency VLBI that is less affected by the foreground material are crucial. Indeed, a recent $86 \mathrm{GHz}$ GMVA polarimetric analysis has detected polarized signals towards the core that might be associated with $B$-fields in the jet formation scales [108]. Also, a KVN $129 \mathrm{GHz}$ monitoring program have revealed multiple $\gamma$-ray emission sites (both the core and C3) thanks to the improved radio- $\gamma$-ray correlation than at lower radio frequencies [104]. Ultimately, these characteristics at high frequencies would render $3 \mathrm{C} 84$ an interesting target for future EHT $230 \mathrm{GHz}$ observations.

\subsection{Other Radio Galaxies}

Other than M87 and 3C 84, an increasing number of nearby $(z<0.1)$ radio galaxies have recently been probed with high-resolution VLBI, resolving their innermost jet geometry. This includes sources such as NGC 4261 [109,110], NGC 6251 [111], NGC 1052 [112-114], Cygnus A [115-117], Centaurus A [118-120], 3C 111 [121,122], 3C 120 [122-124], 3C 264 [125], and PKS 1514+00 [122]. Remarkably, all of these jets show parabolic (or possibly cylindrical for NGC 1052 [114]) shapes at pc-subpc scales, indicating that the collimation zone is well resolved in these radio galaxies. Moreover, 6 out of these jets (NGC 4261, NGC 6251, NGC 1052, 3C 111, 3C 120, and PKS 1514+00) show parabolic (cylindrical) to conical transitions near the sphere of gravitational influence of SMBH, akin to M87, implying a common jet collimation mechanism being at work in these sources.

In contrast, the Cygnus A jet keeps a parabolic shape from 0.3 pc to $60 \mathrm{kpc}$ [117]. This is remarkable since the jet collimation is sustained over 8 orders of magnitude in gravitational radii, well beyond the gravitational influence of the central BH. As Cygnus A is a powerful FR-II jet while the other sources with jet break are mostly FR-I (except for 3C 111), this implies that a high power jet can drill into the ambient medium with its shape kept intact [126]. Alternatively the Cygnus A jet could be pressure balanced against the ambient medium for such large scales due to the cocoon or backflow from the hotspot surrounding the large-scale jet, which is unique to FR-II sources. To better elucidate the possible dichotomy in jet collimation shape between FR-I and FR-II, it would be necessary to increase the size of the FR-II sample.

Another remarkable update on radio galaxies from recent MWL observations is that a growing number of radio galaxies have been detected, even in $\mathrm{TeV} \gamma$-rays, which is surprising given the misaligned nature of this population. To date, six $\mathrm{TeV}$ emitting radio galaxies are identified (Cen $\mathrm{A}$, M87, 3C 84, IC 310, 3C 264 and PKS 0625-35), and some of them (3C 84, IC 310 and M87) show extremely fast $\mathrm{TeV} \gamma$-ray variability ( $\Delta t<1$ day), thus implying very compact emitting scales. As demonstrated in M87, coordinated VLBI monitoring of the nuclei along with $\mathrm{TeV}$ instruments would be very useful to better constrain the site of active emission. For more details on this topic see reviews in $[85,127]$.

\section{Blazars}

Blazars are the most extreme class of AGN, in which the jet axis is almost aligned with our line-of-sight $\left(i<10^{\circ}\right)$. They are further divided into flat spectrum radio quasars (FSRQ) and BL Lac objects based on different optical emission line properties [2]. In the 1990s, virtually all the $\gamma$-ray AGN detected by the Energetic Gamma Ray Experiment Telescope (EGRET) on board the Compton Gamma 
Ray Observatory (CGRO) were classified as blazars [128], and to date more than $3000 \gamma$-ray sources are identified as (or associated with) blazars [24] thanks to the Fermi Large Area Telescope (LAT). Broadband spectral energy distributions (SED) from radio to $\gamma$-rays of blazars are dominated by highly Doppler-boosted nonthermal radiation from the beamed jet, as characterized by a two two-humped shape in the $v-v F_{v}$ domain (synchrotron and inverse Compton emission in the low/high-energy sides, respectively). The boosted emission accompanies frequent flares or rapid variability in all wave bands, offering an excellent opportunity to study the physics of relativistic jets, their internal structures, and flaring mechanisms. The bright and compact nature of blazars on pc scales makes them a dominant target population of VLBI studies since the early days of VLBI astronomy. Nevertheless, a number of fundamental questions on blazars remain to be resolved. Here we highlight some recent progress of VLBI studies on blazars.

\subsection{Parsec-Scale Jets in Blazars: Statistical Properties}

The largest statistical studies on the pc-scale jets of blazars have been performed in the Monitoring of Jets in Active Galactic Nuclei With VLBA Experiments (MOJAVE) program (see, e.g., in $[129,130]$ ). MOJAVE monitors a large number of AGN jets using VLBA at $15 \mathrm{GHz}$ on a monthly basis, with the primary purpose to investigate the mas-scale (typically $~ 1-100$ pc projected) structures of bright jets and their structural evolution, as well as to search for the connection to the observed jet properties at other wavelengths. The MOJAVE program, together with its precursor the VLBA $2 \mathrm{~cm}$ Survey [131], is a roughly 20-year and still ongoing long-term program and a total of $>400$ sources (mostly blazars) have been monitored so far, whereas $\sim 200$ selected sources are currently monitored.

Thanks to the large accumulation of jet monitoring data over two decades, the program now determines the proper motion and its time derivative (i.e., acceleration) of individual jet features in great detail. A majority of jet features show a trend of radial acceleration within deprojected distances $\sim 100 \mathrm{pc}$ from the core, while beyond which the jet components begin to decelerate or remain nearly constant in speed [132-134]. This suggests that the jet acceleration zone of blazars are quite extended. The program also confirmed that a number of jets $(>40)$ contain quasi-stationary features particularly within a few parsecs (projected) from the core [134], which might be associated with recollimation shocks. Especially in BL Lacertae, Cohen et al. [135] showed that a stationary feature at $0.34 \mathrm{pc}$ (projected) from the core behaves as a nozzle ejecting superluminal knots, which is very similar to HST-1 in M87.

The accumulated jet images for each source also reveal time-averaged but ultra-deep maps for each jet by stacking multi-epoch images. Based on stacked MOJAVE images for $>300$ jets, Pushkarev et al. [136] found that the typical jet geometry in their samples is close to conical on scales of 100-1000 pc, even when the jet appears knotty at individual epochs. This indicates that in most jets the underlying flow channels are much wider than previously thought. On smaller scales, in contrast, a number of jets show quasi-parabolic shapes. Interestingly, the jets with significant radial accelerated motion undergo more active collimation, implying the coexistence of acceleration and collimation.

MOJAVE also found that the above jet collimation/acceleration statistics of blazars seem to be closely linked to their $\gamma$-ray properties. The LAT-detected $\gamma$-ray AGN in the MOJAVE sample tend to have higher jet speeds than non-LAT-detected AGN [133]. Also, the LAT-detected sources tend to have narrower intrinsic opening angles and smaller viewing angles than non-LAT-detected jets [136]. This strongly suggests that the observed $\gamma$-ray luminosity of blazars is controlled by pc-scale jet Lorentz factor, viewing angle and opening angle, confirming the Doppler beaming scenario [137].

These pc-scale statistical properties of blazar jets found by MOJAVE are largely consistent with those found by another famous blazar program led by the Boston University (BU), which is regularly monitoring over 30 of the brightest $\gamma$-ray blazars with VLBA at $43 \mathrm{GHz}$ [138]. The BU program often reveals more remarkable correlation between pc-scale jets and $\gamma$-ray events in terms of structural changes (i.e., superluminal ejections) and light curves than at $15 \mathrm{GHz}$. This indicates that the $\gamma$-ray 
production site of blazars is more closely associated with the mm-wave core which is closer to the $\mathrm{BH}$, motivating higher-resolution studies towards the jet base as described in the next subsection.

Based on MOJAVE samples another notable finding is reported by Zamaninasab et al. [139] (Figure 5). They found in 76 MOJAVE sources (including eight radio galaxies) that the poloidal magnetic fluxes threading pc-scale jets (estimated via core-shift) are in nice agreement with the theoretical magnetic fluxes threading the $\mathrm{BH}$ horizon as expected from the magnetically arrested disks (MAD) [5], meaning that many powerful blazar jets are generated via strong ordered $B$-fields anchored by a spinning BH. This, again, stimulates higher-resolution VLBI dissecting the deepest part of blazar jets and their $B$-fields.

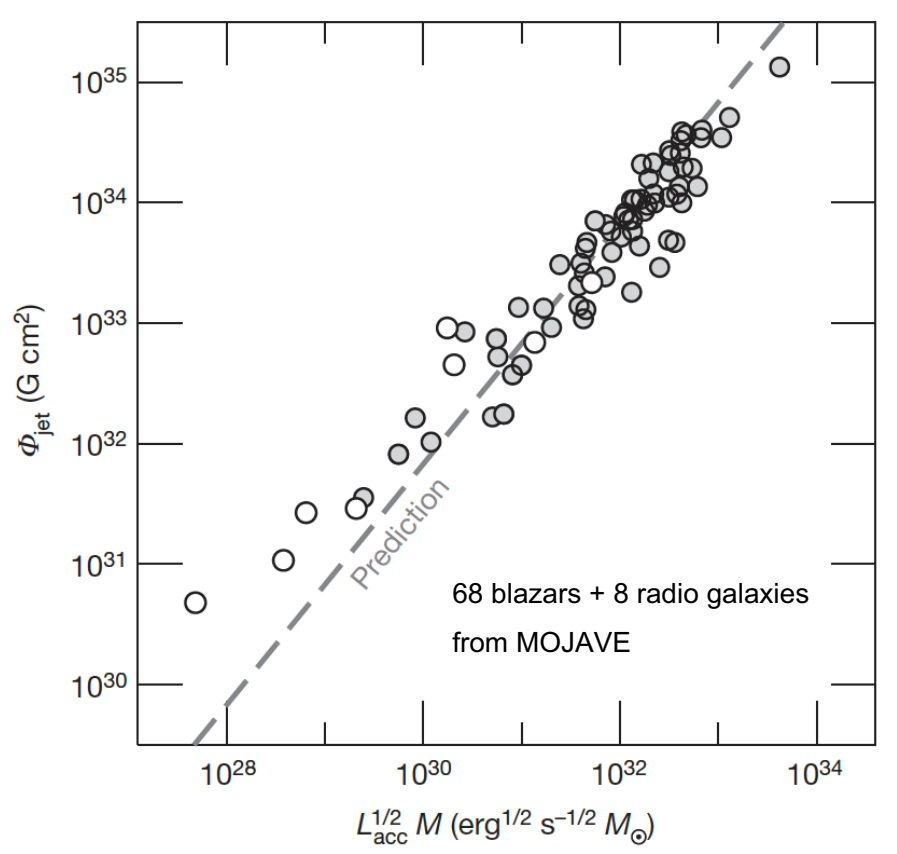

Figure 5. Magnetic flux measured in pc-scale jets via core-shift ( $\left.\Phi_{\text {jet }}\right)$ for a sample of 76 MOJAVE sources (68 blazars (filled circles) and 8 radio galaxies (open circles)), plotted as a function of $L_{\text {acc }}^{1 / 2} M$ that is proportional to the theoretical MAD magnetic flux threading $\mathrm{BH}\left(\Phi_{\mathrm{BH}}\right)$ and is estimated by the accretion disk luminosity $\left(L_{\mathrm{acc}}\right)$ and $\mathrm{BH}$ mass $(M)$ (adapted from the work in [139]).

\subsection{The Innermost Regions of Blazars}

The innermost regions of blazars are often probed by VLBI at $\geq 22 \mathrm{GHz}$. Thanks to its higher angular resolution and transparency to the jet base, high-frequency VLBI can resolve jet structures of blazars on pc-subpc scales, and the connection to the jet properties observed in optical $/ X$-ray $/ \gamma$-ray bands can be better examined.

The most prominent feature in the innermost part of blazars is the radio core at the apparent root of VLBI jet images. The physical origin of the blazar radio core and its location with respect to the central $\mathrm{BH}$ have been debated in the past decade and it is still a lively question at present. Dense VLBI monitoring at $22 / 43 \mathrm{GHz}$, in the last decade, discovered a number of blazars ejecting of superluminal components from the core near in time to active $\gamma$-ray flares, and some of the events further accompany systematic rotation of optical/millimeter polarization angles (see, e.g., in [140-144]. These results suggest that the core of blazars represents a stationary recollimation shock at some distances (typically between $\sim 0.1$ and $\sim 20 \mathrm{pc}$ ) from the $\mathrm{BH}$, likely appearing at the end of ACZ. This seems to be in contrast to the case of the core in radio galaxies, which is likely a synchrotron-self-absorbed $\left(\tau_{\text {ssa }} \sim 1\right)$ surface near the BH within ACZ (e.g., [33,109,112]).

For a few selected blazars, finer-scale structures in the core region are resolved thanks to ultrahigh angular resolution imaging with RadioAstron [145-149]. An excellent example is BL Lacertae by 
Gomez et al. [146], where the jet base was imaged at $22 \mathrm{GHz}$ at $21 \mu$ as resolution. The image revealed the emission upstream of the radio core. This indicates that the upstream emission originates near the $\mathrm{BH}$, while the radio core marks a recollimation shock formed at $\sim 40 \mu$ as $(0.05$ pc projected) from the jet apex. Furthermore, their spatially-resolved polarimetry within 0.5 mas of the core revealed significant gradients in RM and EVPA as a function of position angle with respect to the core, providing evidence that the jet is threaded by helical $B$-fields at these scales (Figure 6 left). These spatially-resolved results confirmed the scenario proposed in the previous MWL observations [140].
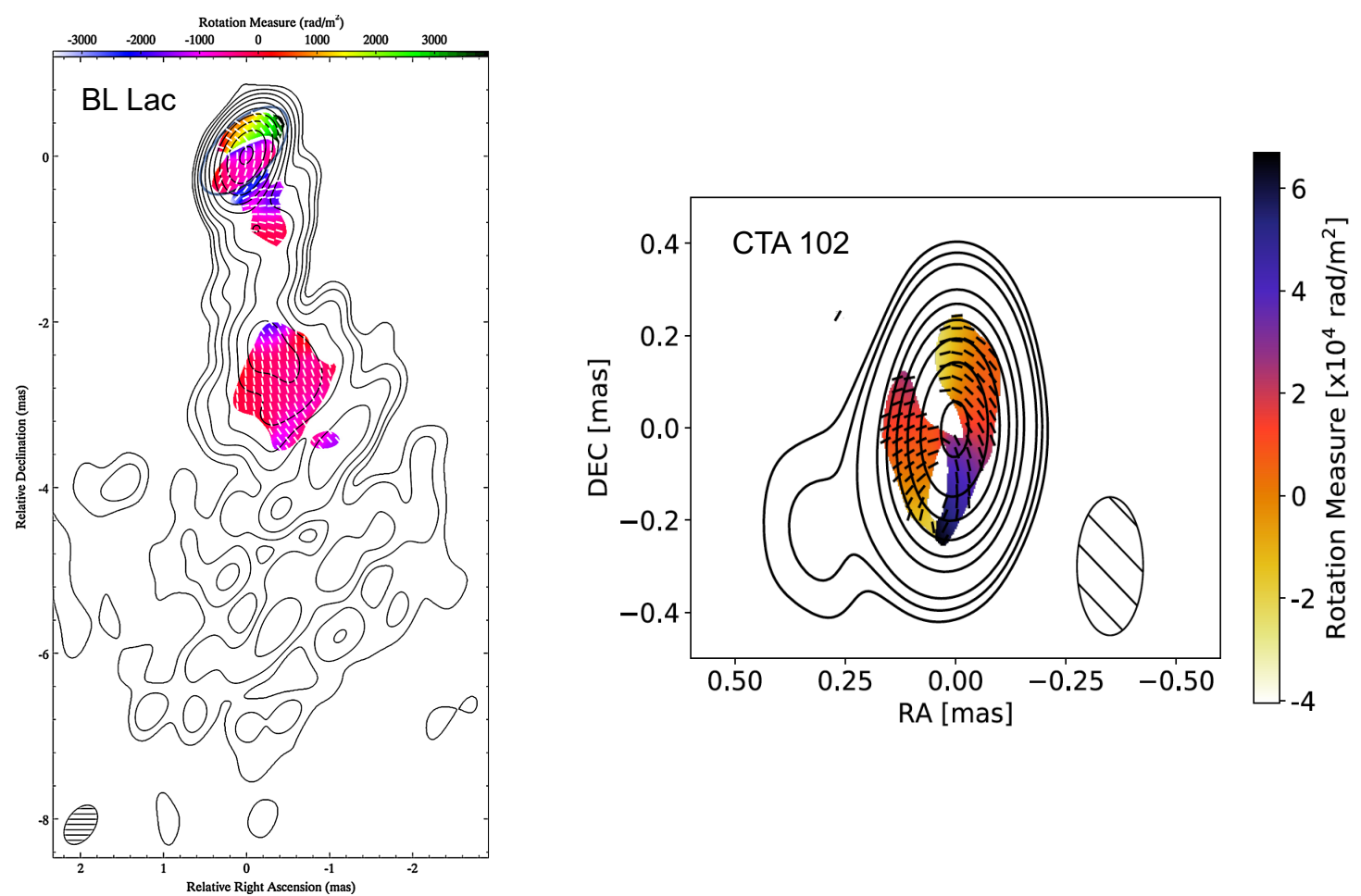

Figure 6. (Left) RM distributions (color) of BL Lacertae made by 15/22/43 GHz data, overlaid on a RadioAstron $22 \mathrm{GHz}$ total intensity contour map (adapted from [146]). (Right) RM map of CTA 102 made by $43 / 86 \mathrm{GHz}$ data, overlaid on a $86 \mathrm{GHz}$ GMVA total intensity contour map (adapted from work in [150]). Both images show significant RM gradients around the core.

In the higher-frequency regime, $86 \mathrm{GHz}$ VLBI reveal features at the core that were not well traced at $22 / 43 \mathrm{GHz}$ alone. A growing number of bright blazars are now regularly observed with GMVA (e.g., S5 0716+714 [151], Mrk501 [152,153], CTA 102 [150,154], NRAO150 [155,156], 3C345 [157], OJ287 [158], PKS 1510-089, and more [159]), many of which are among the ongoing targets of the BU-VLBA $43 \mathrm{GHz}$ program. For CTA 102, a combined polarimetric analysis of GMVA $86 \mathrm{GHz}$ and VLBA $43 \mathrm{GHz}$ data revealed a significant RM gradient around the core [150] (Figure 6 right), similarly to the case of BL Lac, implying the presence of twisted $B$-fields at $\sim 1$ pc scales. GMVA images of the above sources also often indicate that jet bending seems to be common in their innermost jet regions, although the exact physical origin is under debate.

Besides GMVA, the mm-wave core of blazars is currently monitored with KVN in a unique way. Although the array is relatively compact, KVN can do simultaneous $22 / 43 / 86 / 129 \mathrm{GHz}$ imaging with a full-polarimetric capability at mas resolution [160]. A systematic KVN study for a sample of $\gamma$-ray blazars find that the core spectra in their samples systematically become steeper at $86-129 \mathrm{GHz}$, implying that the fully optically thin regime of the jet base is beginning to be accessed at these frequencies [161]. Furthermore, Park et al. [162] have found a systematic increase of RM magnitude with increasing frequency in the mm-wave core of several brightest blazars, indicating a greater $B$-field strength and electron densities towards the jet base. 


\subsection{Core-Shift}

Another powerful method with VLBI to study the innermost regions of relativistic jets is to measure the "core-shift" effect, which is a mas/ $\mu$ as-scale frequency dependent shift of the radio core position. When the radio core at each frequency corresponds to the surface where the optical depth for synchrotron self-absorption (SSA) becomes unity, the position of the radio core moves towards the jet base with increasing frequency [163]. Core-shifts can be measured by either a phase-referencing method (that utilizes an external position calibrator, see e.g., in $[33,164]$ ) or a self-referencing method (that takes advantage of optically-thin jet features in the target itself, see, e.g., in [165-167]). With the core-shift one can constrain/estimate various jet parameters such as the location of the jet apex, innermost jet collimation profile, $B$-field strength and particle number density $[168,169]$. Surveys of the core-shift for a large number of blazars show that most of jets follow a frequency dependence of $r_{\text {core }}(v) \propto v^{-1}[166,167]$ that is consistent with the Blandford \& Königel conical jet model $[163]^{8}$, whereas a recent study combining the results of core-shifts and core sizes for many blazars [170] found that their innermost jet geometries do not exactly follow a pure-conical shape but rather can be described by semiparabolic shapes. This suggests that jet collimation scales are resolved near the core in many blazars, which is consistent with other jet morphology studies [136].

The presence of core-shifts in many blazars seems to be apparently inconsistent with the recollimation shock scenario for blazar cores. One possible idea to accommodate this apparent inconsistency would be that radio cores at low frequencies are SSA-thick surfaces in the downstream side of the jet, whereas high-frequency $(\geq 43 \mathrm{GHz})$ radio cores represent shock features in the upstream side of the jet (see, e.g., in [164]). However, if we recall M87 and HST-1, the above scenario may not always be the case since the standing shock in the downstream side (HST-1) is more pronounced at low frequencies while the radio core at the jet base is in good agreement with a SSA surface up to $43-86 \mathrm{GHz}$ (then the core becomes optically thin at $230 \mathrm{GHz}$ as we see the $\mathrm{BH}$ shadow). Probably other parameters such as jet viewing angles and/or possible jet stratification should be carefully taken into account to properly examine this issue.

\subsection{Polarimetric Properties}

On mas scales, blazars are also bright in polarized flux, in contrast to radio galaxies, in which the pc-scale regions are likely highly depolarized due to the dense foreground medium [171]. As some of the recent highlights are already introduced before, bright polarized signals of blazars give us clues on various internal jet physics such as the global/local structures of $B$-fields, shock compression, velocity shears, as well as the Faraday screen surrounding the jet.

A continuing debate regarding VLBI polarimetry of AGN jets is whether Faraday RM gradients are present transverse to the jet or not. If a jet possesses helical $B$-fields, as is commonly invoked in magnetically-driven models, one should see a systematic RM gradient across the jet because of the gradual change of the $B$-field component along our line-of-sight [172], as demonstrated also in recent dedicated GRMHD simulations [173]. The first observational signature of such a transverse RM gradient was reported in the pc-scale jet of 3C 273 [174]. To date, transverse RM gradients have been reported in $\sim 30$ pc-scale jets (see, e.g., in [165,175-182]). Moreover, as mentioned before, the recent high resolution polarimetric studies with GMVA and RadioAstron have revealed transverse RM gradients even closer to the jet base $[146,150]$, implying that helical $B$-fields in jets are preserved over a wide range of distances from the innermost regions to far beyond the VLBI core. However, the persistence of ordered fields for such long distances would be nontrivial, as the downstream part of the jet beyond the radio core is expected to be largely kinetically dominated, so the $B$-fields would become tangled by

8 It should be noted that a core-shift with $v^{-1}$ could also be reproduced "artificially" by the effect of core-jet blending with limited angular resolution. Such a blending effect would be especially serious for distant jet sources (and in particular at low frequencies where the jet emission is more pronounced and the angular resolution is worse than at high frequencies). 
MHD instabilities. Since most of the previous jet RM images are highly sensitivity limited with patchy RM spots covering only a small fraction of the total jet area, more sensitive VLBI polarimetry at $\mathrm{cm}$ wavelengths, such as the one connected to the Square Kilometer Array (SKA) ${ }^{9}$, would be the key to definitely determine the global $B$-field configuration.

Note that significant progress in blazar polarimetry has also been obtained from radio facilities in $\mathrm{mm} /$ submm bands $(86 / 129 / 230 / 300 \mathrm{GHz})$, thanks to the advent of ALMA as well as KVN/GMVA. In particular, recent $\mathrm{mm}$ / submm RM measurements of blazars consistently show a systematic increase of RM magnitude towards higher frequencies [162,183,184], reaching as high as $10^{5-8} \mathrm{rad} \mathrm{m}^{-2}$, which are at least $\sim 2-5$ orders of magnitude higher than those typically measured at low frequencies. This strongly indicates the existence of dense Faraday screens and/or strong, highly-ordered B-fields towards the jet base, as expected by magnetic-driven jet models. In any case, future $\mathrm{mm} / \mathrm{submm}-\mathrm{VLBI}$ RM measurements are required to spatially resolve the site causing such large RM and thus to reveal the $B$-field structures in the Poynting-dominated regime of the jet.

\section{Narrow-Line Seyfert 1 Galaxies}

One of the most surprising discoveries by the Fermi satellite is the detection of $\gamma$-ray emission from narrow-line Seyfert 1 galaxies (NLSy1s) [185]. NLSy1s are a subclass of AGN defined by its optical properties, i.e., narrow permitted lines of FWHM $(\mathrm{H} \beta<2000) \mathrm{km} \mathrm{s}^{-1}$, $\left[\mathrm{O}_{\mathrm{III}}\right] / \mathrm{H} \beta<3$, and a bump due to $\mathrm{Fe}_{\mathrm{II}}$ emission lines [186] along with a large soft X-ray excess (see, e.g., in [187]). NLSy1s are suggested to have low $\mathrm{BH}$ masses $\left(10^{6-8} \mathrm{M}_{\odot}\right)$ and high accretion rates (near the Eddington limit). While a majority ( $~ 90 \%)$ of them are radio quiet $(R<10)$, about $7 \%$ are radio-loud $(R \geq 10)$ and the remaining $\sim 3 \%$ are very radio-loud $(R>100)$ [188]. The discovery of $\gamma$-ray emission from NLSy1s indicates the emergence of a new class of AGN that can produce powerful relativistic jets in addition to blazars and radio galaxies. To date, 9 sources have securely been confirmed as "bona fide" $\gamma$-ray emitting NLSy1s in the 4FGL catalog [24], whereas some more sources are claimed in Paliya et al. [189], although these additional ones should be regarded as "candidate" $\gamma$-ray NLSy1s due to their less definitive nature (see [190]). Compared to blazars and radio galaxies, the nature of the NLSy1 jets is still poorly understood, i.e., according to the standard AGN paradigm, the combination of low $M_{\mathrm{BH}}$ and high $\dot{M}$ makes it difficult to generate powerful jets. Therefore, the physics of NLSy1 jets requires much more exploration, and high-resolution radio observations may help understand the mechanisms of relativistic jet formation in a possible distinct $\left(M_{\mathrm{BH}}, \dot{M}\right)$ domain from the rest of the jetted AGN classes. Below, we briefly overview recent radio/VLBI studies on (mainly radio-loud) NLSy1s. For an extensive review on $\gamma$-ray emitting NLSy1s and their broadband properties. please see D'Ammando [190].

\subsection{Parsec-Scale Radio Properties of NLSy1s}

So far, approximately 40 NLSy1s have been observed with VLBI (see, e.g., in [191-204]), where most of them are radio-loud and only seven sources are radio quiet [197]. The pc-scale morphology of these sources is predominantly either very compact (point like) or a core with one-sided jet, while a smaller number of sources show two-sided structures. The brightness temperature of the radio core of NLSy1 is typically above $10^{7} \mathrm{~K}$ (with a few sources up to $10^{12} \mathrm{~K}$ ) $[192,197,202]$. These characteristics indicate that the pc-scale radio emission from the radio detected NLSy1s is Doppler beamed, similar to blazars. A blazar-like radio emitting nature of NLSy1s is also confirmed from single-dish studies which found intensive radio variability [201,205,206]. On kpc scales NLSy1s are generally compact at radio, but extended structures ranging from $\sim 1 \mathrm{kpc}$ to $\sim 100 \mathrm{kpc}$ (projected), with a variety of morphology, have recently been detected in an increasing number of sources [207-209].

9 https://www.skatelescope.org/. 
In terms of the kinematic properties of NLSy1 jets, six bright $\gamma$-ray NLSy1s were regularly monitored with MOJAVE [133]. They found significant superluminal motions (up to $\sim 10 c$ ) in three out of the six sources $(1 \mathrm{H} 0323+342$, SBS 0846+513, PMN J0948+0022), suggesting that (at least for the six sources) strong Doppler boosting with small jet viewing angles $\left(i \leq 10^{\circ}\right)$ is at work, similar to blazars. The jet kinematics of other radio-loud NLSy1s (including non- $\gamma$-ray detected ones) are still largely unknown due to the general compactness of the source morphology and the absence of systematic monitoring programs.

\subsection{A Case Study: The Nearest $\gamma$-Ray NLSy1 1H 0323+342}

Among the known $\gamma$-ray emitting NLSy1s, a particularly interesting object would be $1 \mathrm{H} 0323+342$ (see also recent review specific to this source [210]). This source is known as the nearest $(z=0.063)$ $\gamma$-ray detected NLSy1 [185], and is one of the few NLSy1s where the host galaxy is imaged [211]. Various measurements suggest a BH mass of the order of $10^{7} M_{\odot}$ [211-214], although there are still counterarguments [215]. On pc scales, one-sided jet morphology is clearly defined [199] with a transversely-resolved (limb-brightening) jet structure [216] (Figure 7 left). Also, a signature of gradual increase of jet speeds (up to $\sim 9 c$ ) from the core to $\sim 8 \mathrm{pc}$ (projected) down the jet is reported $[133,216,217]$. Thus, the rich source structures along with the proximity offers an excellent opportunity for probing the details of the innermost part of NLSy1 jets at the finest linear scale in all active NLSy1 (1 mas $=1.2 \mathrm{pc})$.

In Hada et al. [218], an extensive morphological analysis on the pc-scale jet of $1 \mathrm{H} 0323+342$ was performed with multi-frequency VLBA data. They discovered that the inner jet morphology is well-characterized by a parabolic (well-collimated) shape, whereas the jet expands more rapidly at larger scales (Figure 7 right). The observed parabolic regions seems to be coincident with those where the jet speed gradually accelerates, suggesting that the jet acceleration and collimation regions are cospatial. Another notable finding in this jet is a bright stationary feature located at 7 mas from the core (corresponding to a deprojected distance $\sim 92 \mathrm{pc}$ for $i=5^{\circ}$ ). The location of the collimation break is coincident with this feature, and it shows highly polarized emission and significant local contraction of jet cross section. These observational characteristics strongly indicate that this feature represents a recollimation shock. Remarkably, these observational features of this jet are overall very similar to those of M87 and HST-1. Although this might suggest a common jet formation mechanism in these sources, such a similarity is surprising given their very different $M_{\mathrm{BH}}, \dot{M}$ and host galaxy types.
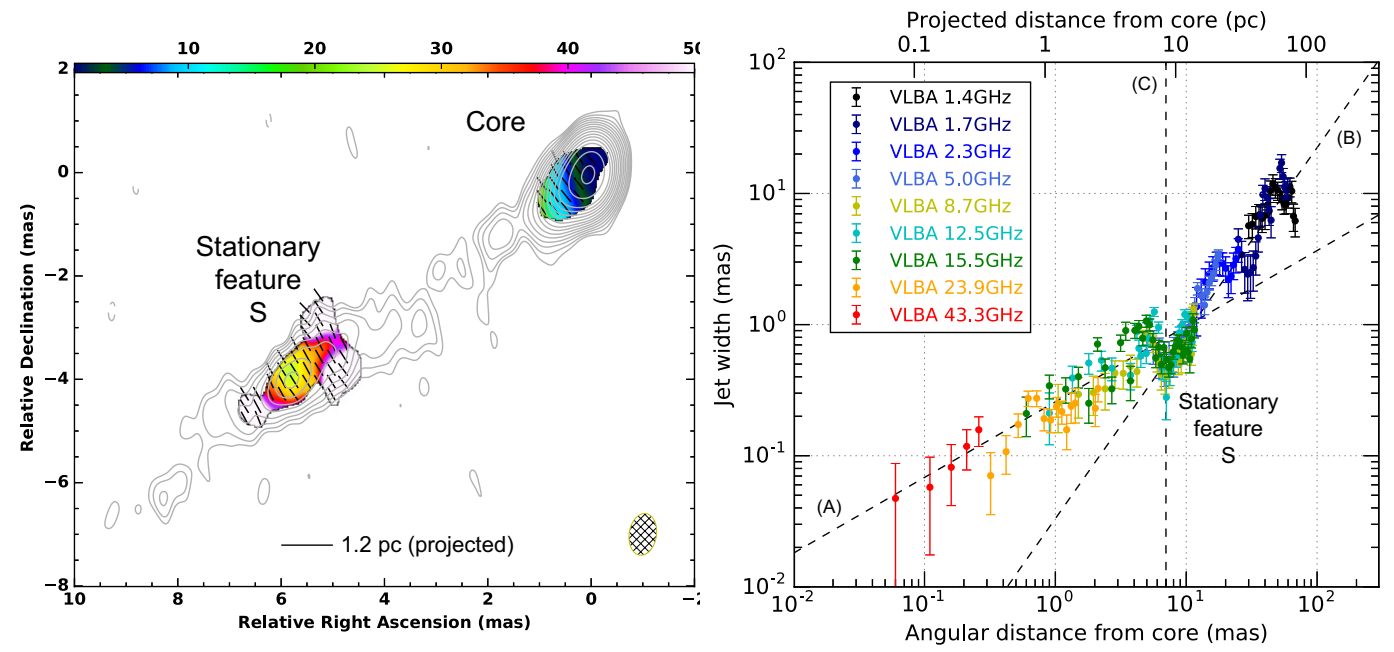

Figure 7. VLBA observations of $1 \mathrm{H} 0323+342$ by [218]. (Left) Contours are $15 \mathrm{GHz}$ total intensity distributions while color scales are polarized fluxes. (Right) Jet width profile as a function of distance from core, showing a parabolic to conical transition at 7 mas (8 pc projected) where the stationary, compressed and polarized feature $S$ is located. Figures adapted from work in [218]. 


\subsection{MWL Properties}

The accumulation of MWL data on $\gamma$-ray NLSy1s reveals that their broadband SEDs are similar to those of FSRQ $[219,220]$, where the overall radiation output is dominated by the inverse Compton component. This indicates the presence of strong external seed photon fields, most likely from the broad-line region or dusty torus illuminated by the luminous accretion flows. However, one of the past $\gamma$-ray events in $1 \mathrm{H} 0323+342$ showed an interesting coincidence with the passage of a superluminal knot through the stationary feature at 7 mas [216]. If these two are physically linked, this implies the production of $\gamma$-rays at an even farther distance from the $\mathrm{BH}\left(\sim 92 \mathrm{pc}\right.$ for $i=5^{\circ}$ : much outside the broad line region (BLR), but likely within the narrow line region (NLR) [210]). Radiation-MHD simulations of super-critical accretion flows suggest the presence of powerful winds driven by strong radiation pressure (see, e.g., in [221]), so such components unique to this type of $\mathrm{BH}$ might help collimate and create a recollimation shock at such far distances.

Due to the similarity to FSRQ together with lower $M_{\mathrm{BH}}$ and higher $\dot{M}$, there is an interesting proposal that radio-loud NLSy1s are at an early evolutionary phase that would have them eventually grow into FSRQ [222,223]. Some past studies suggest that radio-loud NLSy1s share some common properties with compact steep spectrum (CSS) objects (see, e.g., in [222], a class of young radio sources. To better understand how NLSy1s fit into the unification and evolutionary scenarios of AGN, further broadband studies would be required. As no NLSy1s have yet been detected in $\mathrm{TeV} \gamma$-rays, forthcoming Cherenkov Telescope Array (CTA) observations of NLSy1s in concert with radio/VLBI would be especially useful to better clarify the connection to other jetted AGN as well as the physics of NLSy1 jets.

\section{Conclusions and Future Prospects}

In this review, we summarized the recent progress of VLBI studies on AGN jets. Here, we summarize what we have discovered from the VLBI results and list some key questions to be addressed with future observations.

- Jet launching scales: As demonstrated by the successful BH shadow imaging for M87, regular operation of EHT $(230 \mathrm{GHz})$ and also GMVA $(86 \mathrm{GHz})$ connected to ALMA has opened up a new frontier on AGN jet studies thanks to their extreme angular resolution of $\sim 50 R_{\mathrm{S}}$ down to a few $R_{\mathrm{S}}$ for nearby sources. The joint use of the mm-VLBI and conventional $\mathrm{cm}-\mathrm{VLBI}$ (sensitive to the large-scale emission) would be particularly interesting as a next step. Such high-resolution, multi-frequency analysis will, for the first time, connect jet images from the central engine to further out thoroughly, which will then permit us to specify the initial jet-launching geometry with subsequent collimation, particle energetics via spatially-resolved spectra, seeds of superluminal knots, and $B$-field strength/configurations in the magnetically-dominated regions.

- Jet ACZ and recollimation shocks: Although Doppler-beamed properties (e.g., $\gamma$-ray emission) are obviously different between radio galaxies and blazars/NLSy1s (i.e., different jet orientations), the accumulated VLBI results are uncovering that a number of jets share common characteristics in terms of (deprojected) geometrical and kinematic properties at innermost scales, i.e., acceleration, collimation, recollimation shocks with structural transitions at the end of $A C Z$, and possibly also helical $B$-fields. It is remarkable that these features are commonly seen in all three types of jetted AGN (radio galaxies, blazars and NLSy1s), suggesting that a common jet formation mechanism is at work over a wide area in the $\left(M_{\mathrm{BH}}, \dot{M}\right)$ domain. However, the dependence of those properties on jet power is still less defined (i.e., FR-I vs FR-II or BL Lac vs FSRQ).

- Radio-TeV connection: Although we have gained a wealth of knowledge about the correlation between radio/VLBI and GeV $\gamma$-rays in AGN jets (especially blazars) thanks to the success of Fermi, the connection between radio and $\mathrm{TeV} \gamma$-rays is still not well understood, due to the 
poorer statistics in $\mathrm{TeV}$ bands than in $\mathrm{GeV}$ [224]. So far only $\sim 60 \mathrm{TeV}$ AGN have been known ${ }^{10}$ despite the continued efforts by the existing $\mathrm{TeV} \gamma$-ray facilities, and most of the TeV AGN are biased towards high-frequency peaked BL Lacs (HBL). Only a handful of FSRQ/radio galaxies and no NLSy1s have been detected in TeV. The CTA, a forthcoming $\gamma$-ray facility that enhances both angular resolution and sensitivity in $\mathrm{TeV}$ bands, is expected to update the $\mathrm{TeV} \gamma$-ray sky dramatically. Coordinated mm-VLBI/cm-VLBI observations with CTA will be the key to robustly constrain the location and origin of the highest energy photons in AGN.

- Multi-layered (spine-sheath) structure: Now, a number of studies propose that an AGN jet is not a simple homogeneous flow but is composed of multiple layers with different velocities or densities, as represented by the spine-sheath scenario (see, e.g., in [225]). However, there is still little direct observational evidence confirming such multi-layered flows. To address this, a key attempt would be to detect both spine and sheath streamlines (with possible distinct spectral/velocity properties) in a spatially-resolved VLBI jet image, as already hinted in recent high sensitivity images of M87 [55,66]. Future ultrahigh dynamic range radio imaging with SKA or ngVLA ${ }^{11}$ connected to VLBI would greatly enhance the chance to detect such structures in many nearby AGN jets.

- Magnetic-field topology: As mentioned before, a growing number of AGN jets are suggested to possess helical $B$-fields based on RM analysis, implying that such $B$-field structures are common to many jets. Nevertheless, this kind of studies may be further updated for a number of aspects. High-frequency VLBI will be able to reveal the $B$-field structures in the magnetically-dominated regions near $\mathrm{BH}$. For low frequencies probing large-scale $B$-fields beyond the VLBI core, again future VLBI together with SKA or ngVLA (a factor of 10 or more enhancement in polarimetric sensitivity) will be the key to unveil RM distributions/gradients over the whole jet area, which is crucial to conclude the exact $B$-field topology.

- Low-luminosity AGN: Here, we only focused on AGN with prominent jets. However, a majority of AGN are radio quiet or even though they are formally radio-loud, the jets are very weak on mas scales (see, e.g., in [226,227]). An extreme example would be the Galactic Center SgrA* (see, e.g., in [228]). To constrain the regulation of jet power and production efficiency in AGN, horizon-scale comparison between powerful/weak-jet AGN would be necessary since GRMHD theories (for RIAF) predict that the magnetic flux threading $\mathrm{BH}$ and the $\mathrm{BH}$ spin are the key parameters for the jet production efficiency [5], both of which are horizon-scale quantities. Nearby LLAGN such as M81 [229], M84 [230], the Sombrero galaxy [231] may be good potential targets of future horizon-scale weak-jet imaging with EHT. LLAGN jets would also be important for understanding the kinetic feedback in galaxy-AGN coevolution, as they could be less collimated than powerful jets so their large solid angles could efficiently heat the host galaxies [232].

- Multi-messanger view: Blazars have long been considered as potential sources of high-energy neutrinos. Recent multi-messenger observations of a blazar TXS 0506+056 discovered a coincidence of $\gamma$-ray flares with high-energy neutrino IceCube-170922A [233], opening a new era on multimessenger astronomy on AGN jets. Analysis of contemporaneous radio and VLBI data on this source found a large flux increase from the radio core that might be associated with the neutrino event [234]. Therefore, future dedicated VLBI follow-up of neutrino candidate AGN would help localize the site of high-energy neutrinos in relativistic jets.

Funding: This research was funded by JSPS KAKENHI (grant number 18KK0090, 19H01943, 18H03721).

Acknowledgments: K.H. thanks the anonymous referees for their careful review of the manuscript. K.H. also acknowledges F. D'Ammando, M. Nakamura, M. Kino, J. Park and Y. Cui for their fruitful comments that improved the manuscript. K.H. is also grateful to L. Loinard for a careful reading of the revised draft.

10 See TeVCat website (http:/ / tevcat.uchicago.edu/).

11 https://ngvla.nrao.edu/. 
Conflicts of Interest: The author declares no conflicts of interest.

\section{References}

1. Rees, M.J. Black Hole Models for Active Galactic Nuclei. Annu. Rev. Astron. Astrophys. 1984, 22, 471-506. [CrossRef]

2. Urry, C.M.; Padovani, P. Unified Schemes for Radio-Loud Active Galactic Nuclei. Publ. Astron. Soc. Pac. 1995, 107, 803. [CrossRef]

3. Curtis, H.D. Descriptions of 762 Nebulae and Clusters Photographed with the Crossley Reflector. Publ. Lick Obs. 1918, 13, 9-42.

4. McKinney, J.C. General relativistic magnetohydrodynamic simulations of the jet formation and large-scale propagation from black hole accretion systems. Mon. Not. R. Astron. Soc. 2006, 368, 1561-1582. [CrossRef]

5. Tchekhovskoy, A.; Narayan, R.; McKinney, J.C. Efficient generation of jets from magnetically arrested accretion on a rapidly spinning black hole. Mon. Not. R. Astron. Soc. 2011, 418, L79-L83. [CrossRef]

6. Marscher, A.P.; Gear, W.K. Models for high-frequency radio outbursts in extragalactic sources, with application to the early 1983 millimeter-to-infrared flare of 3C 273. Astrophys. J. 1985, 298, 114-127. [CrossRef]

7. Sikora, M.; Begelman, M.C.; Rees, M.J. Comptonization of Diffuse Ambient Radiation by a Relativistic Jet: The Source of Gamma Rays from Blazars? Astrophys. J. 1994, 421, 153. [CrossRef]

8. Spada, M.; Ghisellini, G.; Lazzati, D.; Celotti, A. Internal shocks in the jets of radio-loud quasars. Mon. Not. R. Astron. Soc. 2001, 325, 1559-1570. [CrossRef]

9. Stawarz, Ł.; Ostrowski, M. Radiation from the Relativistic Jet: A Role of the Shear Boundary Layer. Astrophys. J. 2002, 578, 763-774. [CrossRef]

10. Giannios, D. Reconnection-driven plasmoids in blazars: Fast flares on a slow envelope. Mon. Not. R. Astron. Soc. 2013, 431, 355-363. [CrossRef]

11. Blandford, R.D.; Znajek, R.L. Electromagnetic extraction of energy from Kerr black holes. Mon. Not. R. Astron. Soc. 1977, 179, 433-456. [CrossRef]

12. Blandford, R.D.; Payne, D.G. Hydromagnetic flows from accretion disks and the production of radio jets. Mon. Not. R. Astron. Soc. 1982, 199, 883-903. [CrossRef]

13. Komissarov, S.S.; Barkov, M.V.; Vlahakis, N.; Königl, A. Magnetic acceleration of relativistic active galactic nucleus jets. Mon. Not. R. Astron. Soc. 2007, 380, 51-70. [CrossRef]

14. Readhead, A.C.S.; Cohen, M.H.; Blandford, R.D. A jet in the nucleus of NGC6251. Nature 1978, 272, $131-134$. [CrossRef]

15. Pearson, T.J.; Unwin, S.C.; Cohen, M.H.; Linfield, R.P.; Readhead, A.C.S.; Seielstad, G.A.; Simon, R.S.; Walker, R.C. Superluminal expansion of quasar 3C273. Nature 1981, 290, 365-368. [CrossRef]

16. Pearson, T.J.; Readhead, A.C.S. The milli-arcsecond structure of a complete sample of radio sources. I. VLBI maps of seven sources. Astrophys. J. 1981, 248, 61-81. [CrossRef]

17. Cohen, M.H.; Pearson, T.J.; Readhead, A.C.S.; Seielstad, G.A.; Simon, R.S.; Walker, R.C. Superluminal variations in 3C 120, 3C 273, and 3C 345. Astrophys. J. 1979, 231, 293-298. [CrossRef]

18. An, T.; Sohn, B.W.; Imai, H. Capabilities and prospects of the East Asia Very Long Baseline Interferometry Network. Nat. Astron. 2018, 2, 118-125. [CrossRef]

19. Hirabayashi, H.; Hirosawa, H.; Kobayashi, H.; Murata, Y.; Edwards, P.G.; Fomalont, E.B.; Fujisawa, K.; Ichikawa, T.; Kii, T.; Lovell, J.E.J.; et al. Overview and Initial Results of the Very Long Baseline Interferometry Space Observatory Programme. Science 1998, 281, 1825. [CrossRef]

20. Kardashev, N.S.; Khartov, V.V.; Abramov, V.V.; Avdeev, V.Y.; Alakoz, A.V.; Aleksandrov, Y.A.; Ananthakrishnan, S.; Andreyanov, V.V.; Andrianov, A.S.; Antonov, N.M.; et al. "RadioAstron"—A telescope with a size of 300,000 km: Main parameters and first observational results. Astron. Rep. 2013, 57, 153-194. [CrossRef]

21. Blandford, R.; Meier, D.; Readhead, A. Relativistic Jets from Active Galactic Nuclei. Annu. Rev. Astron. Astrophys. 2019, 57, 467-509. [CrossRef]

22. The Fermi-LAT Collaboration. The Fourth Catalog of Active Galactic Nuclei Detected by the Fermi Large Area Telescope. arXiv 2019, arXiv:1905.10771.

23. Fanaroff, B.L.; Riley, J.M. The morphology of extragalactic radio sources of high and low luminosity. Mon. Not. R. Astron. Soc. 1974, 167, 31P-36P. [CrossRef]

24. The Fermi-LAT Collaboration. Fermi Large Area Telescope Fourth Source Catalog. arXiv 2019, arXiv:1902.10045. 
25. Blakeslee, J.P.; Jordán, A.; Mei, S.; Côté, P.; Ferrarese, L.; Infante, L.; Peng, E.W.; Tonry, J.L.; West, M.J. The ACS Fornax Cluster Survey. V. Measurement and Recalibration of Surface Brightness Fluctuations and a Precise Value of the Fornax-Virgo Relative Distance. Astrophys. J. 2009, 694, 556-572. [CrossRef]

26. Owen, F.N.; Hardee, P.E.; Cornwell, T.J. High-resolution, high dynamic range VLA images of the M87 jet at 2 centimeters. Astrophys. J. 1989, 340, 698. [CrossRef]

27. Biretta, J.A.; Sparks, W.B.; Macchetto, F. Hubble Space Telescope Observations of Superluminal Motion in the M87 Jet. Astrophys. J. 1999, 520, 621-626. [CrossRef]

28. Perlman, E.S.; Biretta, J.A.; Sparks, W.B.; Macchetto, F.D.; Leahy, J.P. The Optical-Near-Infrared Spectrum of the M87 Jet fromHubble Space Telescope Observations. Astrophys. J. 2001, 551, 206-222. [CrossRef]

29. Harris, D.E.; Cheung, C.C.; Biretta, J.A.; Sparks, W.B.; Junor, W.; Perlman, E.S.; Wilson, A.S. The Outburst of HST-1 in the M87 Jet. Astrophys. J. 2006, 640, 211-218. [CrossRef]

30. Macchetto, F.; Marconi, A.; Axon, D.J.; Capetti, A.; Sparks, W.; Crane, P. The Supermassive Black Hole of M87 and the Kinematics of Its Associated Gaseous Disk. Astrophys. J. 1997, 489, 579-600. [CrossRef]

31. Walsh, J.L.; Barth, A.J.; Ho, L.C.; Sarzi, M. The M87 black hole mass from gas-dynamical models of space telescope imaging spectrograph observations. Astrophys. J. 2013, 770, 86. [CrossRef]

32. Gebhardt, K.; Adams, J.; Richstone, D.; Lauer, T.R.; Faber, S.M.; Gültekin, K.; Murphy, J.; Tremaine, S. The Black Hole Mass in M87 from Gemini/NIFS Adaptive Optics Observations. Astrophys. J. 2011, 729, 119. [CrossRef]

33. Hada, K.; Doi, A.; Kino, M.; Nagai, H.; Hagiwara, Y.; Kawaguchi, N. An origin of the radio jet in M87 at the location of the central black hole. Nature 2011, 477, 185-187. [CrossRef] [PubMed]

34. Doeleman, S.S.; Fish, V.L.; Schenck, D.E.; Beaudoin, C.; Blundell, R.; Bower, G.C.; Broderick, A.E.; Chamberlin, R.; Freund, R.; Friberg, P.; et al. Jet-Launching Structure Resolved Near the Supermassive Black Hole in M87. Science 2012, 338, 355-358. [CrossRef] [PubMed]

35. Akiyama, K.; Lu, R.S.; Fish, V.L.; Doeleman, S.S.; Broderick, A.E.; Dexter, J.; Hada, K.; Kino, M.; Nagai, H.; Honma, M.; et al. $230 \mathrm{GHz}$ VLBI observations of M87: Event-horizon-scale structure during an enhanced very-high-energy $\gamma$-ray state in 2012. Astrophys. J. 2015, 807, 150. [CrossRef]

36. Broderick, A.E.; Loeb, A. Imaging the Black Hole Silhouette of M87: Implications for Jet Formation and Black Hole Spin. Astrophys. J. 2009, 697, 1164-1179. [CrossRef]

37. The Event Horizon Telescope Collaboration; Akiyama, K.; Alberdi, A.; Alef, W.; Asada, K.; Azulay, R.; Baczko, A.K.; Ball, D.; Balokovic, M.; Barrett, J.; et al. First M87 Event Horizon Telescope Results. I. The Shadow of the Supermassive Black Hole. Astrophys. J. Lett. 2019, 875, L1. [CrossRef]

38. The Event Horizon Telescope Collaboration; Akiyama, K.; Alberdi, A.; Alef, W.; Asada, K.; Azulay, R.; Baczko, A.K.; Ball, D.; Balokovic, M.; Barrett, J.; et al. First M87 Event Horizon Telescope Results. II. Array and Instrumentation. Astrophys. J. Lett. 2019, 875, L2. [CrossRef]

39. The Event Horizon Telescope Collaboration; Akiyama, K.; Alberdi, A.; Alef, W.; Asada, K.; Azulay, R.; Baczko, A.K.; Ball, D.; Balokovic, M.; Barrett, J.; et al. First M87 Event Horizon Telescope Results. III. Data Processing and Calibration. Astrophys. J. Lett. 2019, 875, L3. [CrossRef]

40. The Event Horizon Telescope Collaboration; Akiyama, K.; Alberdi, A.; Alef, W.; Asada, K.; Azulay, R.; Baczko, A.K.; Ball, D.; Balokovic, M.; Barrett, J.; et al. First M87 Event Horizon Telescope Results. IV. Imaging the Central Supermassive Black Hole. Astrophys. J. Lett. 2019, 875, L4. [CrossRef]

41. The Event Horizon Telescope Collaboration; Akiyama, K.; Alberdi, A.; Alef, W.; Asada, K.; Azulay, R.; Baczko, A.K.; Ball, D.; Balokovic, M.; Barrett, J.; et al. First M87 Event Horizon Telescope Results. V. Physical Origin of the Asymmetric Ring. Astrophys. J. Lett. 2019, 875, L5. [CrossRef]

42. The Event Horizon Telescope Collaboration; Akiyama, K.; Alberdi, A.; Alef, W.; Asada, K.; Azulay, R.; Baczko, A.K.; Ball, D.; Balokovic, M.; Barrett, J.; et al. First M87 Event Horizon Telescope Results. VI. The Shadow and Mass of the Central Black Hole. Astrophys. J. Lett. 2019, 875, L6. [CrossRef]

43. Hada, K.; Kino, M.; Doi, A.; Nagai, H.; Honma, M.; Akiyama, K.; Tazaki, F.; Lico, R.; Giroletti, M.; Giovannini, G.; et al. High-sensitivity 86 GHz (3.5 mm) VLBI Observations of M87: Deep Imaging of the Jet Base at a Resolution of 10 Schwarzschild Radii. Astrophys. J. 2016, 817, 131. [CrossRef]

44. Kim, J.Y.; Lee, S.S.; Hodgson, J.A.; Algaba, J.C.; Zhao, G.Y.; Kino, M.; Byun, D.Y.; Kang, S. Long-term millimeter VLBI monitoring of M87 with KVN at milliarcsecond resolution: Nuclear spectrum. Astron. Astrophys. 2018, 610, L5. [CrossRef] 
45. Mościbrodzka, M.; Falcke, H.; Shiokawa, H. General relativistic magnetohydrodynamical simulations of the jet in M87. Astron. Astrophys. 2016, 586, A38. [CrossRef]

46. Takahashi, K.; Toma, K.; Kino, M.; Nakamura, M.; Hada, K. Fast-spinning Black Holes Inferred from Symmetrically Limb-brightened Radio Jets. Astrophys. J. 2018, 868, 82. [CrossRef]

47. Nakamura, M.; Asada, K.; Hada, K.; Pu, H.Y.; Noble, S.; Tseng, C.; Toma, K.; Kino, M.; Nagai, H.; Takahashi, K.; et al. Parabolic Jets from the Spinning Black Hole in M87. Astrophys. J. 2018, 868, 146. [CrossRef]

48. Chael, A.; Narayan, R.; Johnson, M.D. Two-temperature, Magnetically Arrested Disc simulations of the jet from the supermassive black hole in M87. Mon. Not. R. Astron. Soc. 2019, 486, 2873-2895. [CrossRef]

49. Walker, R.C.; Hardee, P.E.; Davies, F.B.; Ly, C.; Junor, W. The Structure and Dynamics of the Subparsec Jet in M87 Based on 50 VLBA Observations over 17 Years at 43 GHz. Astrophys. J. 2018, 855, 128. [CrossRef]

50. Asada, K.; Nakamura, M. The Structure of the M87 Jet: A Transition from Parabolic to Conical Streamlines. Astrophys. J. 2012, 745, L28. [CrossRef]

51. Hada, K.; Kino, M.; Doi, A.; Nagai, H.; Honma, M.; Hagiwara, Y.; Giroletti, M.; Giovannini, G.; Kawaguchi, N. The Innermost Collimation Structure of the M87 Jet Down to 10 Schwarzschild Radii. Astrophys. J. 2013, 775, 70. [CrossRef]

52. Nakamura, M.; Asada, K. The Parabolic Jet Structure in M87 as a Magnetohydrodynamic Nozzle. Astrophys. J. 2013, 775, 118. [CrossRef]

53. Di Matteo, T.; Allen, S.W.; Fabian, A.C.; Wilson, A.S.; Young, A.J. Accretion onto the Supermassive Black Hole in M87. Astrophys. J. 2003, 582, 133-140. [CrossRef]

54. Russell, H.R.; Fabian, A.C.; McNamara, B.R.; Broderick, A.E. Inside the Bondi radius of M87. Mon. Not. R. Astron. Soc. 2015, 451, 588-600. [CrossRef]

55. Asada, K.; Nakamura, M.; Pu, H.Y. Indication of the Black Hole Powered Jet in M87 by VSOP Observations. Astrophys. J. 2016, 833, 56. [CrossRef]

56. Hada, K. The Structure and Propagation of the Misaligned Jet M87. Galaxies 2016, 5, 2. [CrossRef]

57. Ogihara, T.; Takahashi, K.; Toma, K. A Mechanism for the Triple-ridge Emission Structure of AGN Jets. Astrophys. J. 2019, 877, 19. [CrossRef]

58. Park, J.; Hada, K.; Kino, M.; Nakamura, M.; Hodgson, J.; Ro, H.; Cui, Y.; Asada, K.; Algaba, J.C.; Sawada-Satoh, S.; et al. Kinematics of the M87 jet in the collimation zone: Gradual acceleration and velocity stratification. arXiv 2019, arXiv:1911.02279.

59. Meyer, E.T.; Sparks, W.B.; Biretta, J.A.; Anderson, J.; Sohn, S.T.; van der Marel, R.P.; Norman, C.; Nakamura, M. Optical proper motion measurements of the M87 jet: New results from the hubble space telescope. Astrophys. J. 2013, 774, L21. [CrossRef]

60. Reid, M.J.; Biretta, J.A.; Junor, W.; Muxlow, T.W.B.; Spencer, R.E. Subluminal motion and limb brightening in the nuclear jet of M87. Astrophys. J. 1989, 336, 112-120. [CrossRef]

61. Dodson, R.; Edwards, P.G.; Hirabayashi, H. Milliarcsecond-Scale Spectral Properties and Jet Motions in M87. Astrophys. J. 2006, 58, 243-251. [CrossRef]

62. Ly, C.; Walker, R.C.; Junor, W. High-Frequency VLBI Imaging of the Jet Base of M87. Astrophys. J. 2007, 660, 200-205. [CrossRef]

63. Kovalev, Y.Y.; Lister, M.L.; Homan, D.C.; Kellermann, K.I. The Inner Jet of the Radio Galaxy M87. Astrophys. J. Lett. 2007, 668, L27-L30. [CrossRef]

64. Walker, R.C.; Ly, C.; Junor, W.; Hardee, P.J. A VLBA movie of the jet launch region in M87. J. Phys. Conf. Ser. 2008, 131, 012053. [CrossRef]

65. Mertens, F.; Lobanov, A.P.; Walker, R.C.; Hardee, P.E. Kinematics of the jet in M87 on scales of 100-1000 Schwarzschild radii. Astron. Astrophys. 2016, 595, A54. [CrossRef]

66. Hada, K.; Park, J.H.; Kino, M.; Niinuma, K.; Sohn, B.W.; Ro, H.W.; Jung, T.; Algaba, J.C.; Zhao, G.Y.; Lee, S.S.; et al. Pilot KaVA monitoring on the M87 jet: Confirming the inner jet structure and superluminal motions at sub-pc scales. Publ. Astron. Soc. Jpn. 2017, 69, 71. [CrossRef]

67. Prieto, M.A.; Fernández-Ontiveros, J.A.; Markoff, S.; Espada, D.; González-Martín, O. The central parsecs of M87: Jet emission and an elusive accretion disc. Mon. Not. R. Astron. Soc. 2016, 457, 3801-3816. [CrossRef]

68. Kuo, C.Y.; Asada, K.; Rao, R.; Nakamura, M.; Algaba, J.C.; Liu, H.B.; Inoue, M.; Koch, P.M.; Ho, P.T.P.; Matsushita, S.; et al. Measuring mass accretion rate onto the supermassive black hole M87 using Faraday rotation with the Submillimeter Array. Astrophys. J. 2014, 783, L33. [CrossRef] 
69. Park, J.; Hada, K.; Kino, M.; Nakamura, M.; Ro, H.; Trippe, S. Faraday Rotation in the Jet of M87 inside the Bondi Radius: Indication of Winds from Hot Accretion Flows Confining the Relativistic Jet. Astrophys. J. 2019, 871, 257. [CrossRef]

70. Blandford, R.D.; Begelman, M.C. On the fate of gas accreting at a low rate on to a black hole. Mon. Not. R. Astron. Soc. 1999, 303, L1-L5. [CrossRef]

71. Aharonian, F.; Akhperjanian, A.G.; Bazer-Bachi, A.R.; Beilicke, M.; Benbow, W.; Berge, D.; Bernlohr, K.; Boisson, C.; Bolz, O.; Borrel, V.; et al. Fast Variability of Tera-Electron Volt Rays from the Radio Galaxy M87. Science 2006, 314, 1424-1427. [CrossRef] [PubMed]

72. Cheung, C.C.; Harris, D.E.; Stawarz, Ł. Superluminal Radio Features in the M87 Jet and the Site of Flaring TeV Gamma-Ray Emission. Astrophys. J. 2007, 663, L65-L68. [CrossRef]

73. The VERITAS Collaboration; The VLBA 43 GHz M87 Monitoring Team; The H.E.S.S. Collaboration; The MAGIC Collaboration; Acciari, V.A.; Aliu, E.; Arlen, T.; Bautista, M.; Beilicke, M.; Benbow, W.; et al. Radio Imaging of the Very-High-Energy $\gamma$-Ray Emission Region in the Central Engine of a Radio GalaxySupporting Online Material. Science 2009, 325, 444-448. [PubMed]

74. Abramowski, A.; Acero, F.; Aharonian, F.; Akhperjanian, A.G.; Anton, G.; Balzer, A.; Barnacka, A.; Becherini, Y.; Becker, J.; Behera, B.; et al. The 2010 very high energy $\gamma$-ray flare and 10 years of multiwavelength Observations of M87. Astrophys. J. 2012, 746, 151. [CrossRef]

75. Harris, D.E.; Massaro, F.; Cheung, C.C.; Horns, D.; Raue, M.; Stawarz, Ł.; Wagner, S.; Colin, P.; Mazin, D.; Wagner, R.; et al. An experiment to locate the site of tev flaring in M87. Astrophys. J. 2011, 743, 177. [CrossRef]

76. Hada, K.; Kino, M.; Nagai, H.; Doi, A.; Hagiwara, Y.; Honma, M.; Giroletti, M.; Giovannini, G.; Kawaguchi, N. VLBI Observations of the Jet in M87 during the very high energy $\gamma$-ray flare in 2010 April. Astrophys. J. 2012, 760, 52. [CrossRef]

77. Beilicke, M. VERITAS Collaboration. In VERITAS Observations of M87 in 2011/2012; Aharonian, F.A., Hofmann, W., Rieger, F.M., Eds.; American Institute of Physics Conference Series; AIP: Melville, NY, USA, 2012; Volume 1505, pp. 586-589. [CrossRef]

78. Hada, K.; Giroletti, M.; Kino, M.; Giovannini, G.; D'Ammando, F.; Cheung, C.C.; Beilicke, M.; Nagai, H.; Doi, A.; Akiyama, K.; et al. A Strong Radio Brightening at the Jet Base of M87 during the Elevated Very High Energy Gamma-Ray State in 2012. Astrophys. J. 2014, 788, 165. [CrossRef]

79. Neronov, A.; Aharonian, F.A. Production of TeV Gamma Radiation in the Vicinity of the Supermassive Black Hole in the Giant Radio Galaxy M87. Astrophys. J. 2007, 671, 85-96. [CrossRef]

80. Lenain, J.P.; Boisson, C.; Sol, H.; Katarzynski, K. A synchrotron self-Compton scenario for the very high energy $\gamma$-ray emission of the radiogalaxy M87. Astron. Astrophys. 2008, 478, 111-120. [CrossRef]

81. Tavecchio, F.; Ghisellini, G. Spine-sheath layer radiative interplay in subparsec-scale jets and the TeV emission from M87. Mon. Not. R. Astron. Soc. Lett. 2008, 385, L98-L102. [CrossRef]

82. Abdo, A.A.; Ackermann, M.; Ajello, M.; Atwood, W.B.; Axelsson, M.; Baldini, L.; Ballet, J.; Barbiellini, G.; Bastieri, D.; Bechtol, K.; et al. Fermilarge area telescope gamma-ray detection of the radio galaxy M87. Astrophys. J. 2009, 707, 55-60. [CrossRef]

83. Barkov, M.V.; Aharonian, F.A.; Bosch-Ramon, V. Gamma-ray flares from red giant/jet interactions in active galactic nuclei. Astrophys. J. 2010, 724, 1517-1523. [CrossRef]

84. Giannios, D.; Uzdensky, D.A.; Begelman, M.C. Fast TeV variability from misaligned minijets in the jet of M87. Mon. Not. R. Astron. Soc. 2010, 402, 1649-1656. [CrossRef]

85. Rieger, F.M.; Levinson, A. Radio Galaxies at VHE Energies. Galaxies 2018, 6, 116. [CrossRef]

86. Giroletti, M.; Hada, K.; Giovannini, G.; Casadio, C.; Beilicke, M.; Cesarini, A.; Cheung, C.C.; Doi, A.; Krawczynski, H.; Kino, M.; et al. The kinematic of HST-1 in the jet of M87. Astron. Astrophys. 2012, 538, L10. [CrossRef]

87. Hada, K.; Giroletti, M.; Giovannini, G.; Casadio, C.; Beilicke, M.; Cesarini, A.; Cheung, T.; Doi, A.; Gómez, J.L.; Krawczynski, H.; et al. Continuing EVN monitoring of HST-1 in the jet of M87. arXiv 2015, arXiv:1504.01808.

88. Pedlar, A.; Ghataure, H.S.; Davies, R.D.; Harrison, B.A.; Perley, R.; Crane, P.C.; Unger, S.W. The radio structure of NGC 1275. Mon. Not. R. Astron. Soc. 1990, 246, 477.

89. Asada, K.; Kameno, S.; Shen, Z.Q.; Horiuchi, S.; Gabuzda, D.C.; Inoue, M. The Expanding Radio Lobe of 3C 84 Revealed by VSOP Observations. Publ. Astron. Soc. Jpn. 2006, 58, 261-270. [CrossRef] 
90. Abdo, A.A.; Ackermann, M.; Ajello, M.; Asano, K.; Baldini, L.; Ballet, J.; Barbiellini, G.; Bastieri, D.; Baughman, B.M.; Bechtol, K.; et al. Fermi Discovery of Gamma-ray Emission from NGC 1275. Astrophys. J. 2009, 699, 31-39. [CrossRef]

91. Nagai, H.; Suzuki, K.; Asada, K.; Kino, M.; Kameno, S.; Doi, A.; Inoue, M.; Kataoka, J.; Bach, U.; Hirota, T.; et al. VLBI Monitoring of 3C 84 (NGC 1275) in Early Phase of the 2005 Outburst. Publ. Astron. Soc. Jpn. 2010, 62, L11. [CrossRef]

92. Suzuki, K.; Nagai, H.; Kino, M.; Kataoka, J.; Asada, K.; Doi, A.; Inoue, M.; Orienti, M.; Giovannini, G.; Giroletti, M.; et al. Exploring the Central Sub-parsec Region of the $\gamma$-Ray Bright Radio Galaxy 3C 84 with VLBA at $43 \mathrm{GHz}$ in the Period of 2002-2008. Astrophys. J. 2012, 746, 140. [CrossRef]

93. Krabbe, A.; Sams, B.J.I.; Genzel, R.; Thatte, N.; Prada, F. Near infrared imaging spectroscopy of NGC 1275. Astron. Astrophys. 2000, 354, 439-452.

94. Salomé, P.; Combes, F.; Edge, A.C.; Crawford, C.; Erlund, M.; Fabian, A.C.; Hatch, N.A.; Johnstone, R.M.; Sanders, J.S.; Wilman, R.J. Cold molecular gas in the Perseus cluster core. Association with X-ray cavity, $\mathrm{H} \alpha$ filaments and cooling flow. Astron. Astrophys. 2006, 454, 437-445. [CrossRef]

95. Lim, J.; Ao, Y.; Dinh-V-Trung. Radially Inflowing Molecular Gas in NGC 1275 Deposited by an X-Ray Cooling Flow in the Perseus Cluster. Astrophys. J. 2008, 672, 252-265. [CrossRef]

96. O'Dea, C.P.; Dent, W.A.; Balonek, T.J. The 20 year spectral evolution of the radio nucleus of NGC 1275. Astrophys. J. 1984, 278, 89-95. [CrossRef]

97. Walker, R.C.; Dhawan, V.; Romney, J.D.; Kellermann, K.I.; Vermeulen, R.C. VLBA Absorption Imaging of Ionized Gas Associated with the Accretion Disk in NGC 1275. Astrophys. J. 2000, 530, 233-244. [CrossRef]

98. Scharwächter, J.; McGregor, P.J.; Dopita, M.A.; Beck, T.L. Kinematics and excitation of the molecular hydrogen accretion disc in NGC 1275. Mon. Not. R. Astron. Soc. 2013, 429, 2315-2332. [CrossRef]

99. Nagai, H.; Onishi, K.; Kawakatu, N.; Fujita, Y.; Kino, M.; Fukazawa, Y.; Lim, J.; Forman, W.; Vrtilek, J.; Nakanishi, K.; et al. The ALMA Discovery of the Rotating Disk and Fast Outflow of Cold Molecular Gas in NGC 1275. Astrophys. J. 2019, 883, 193. [CrossRef]

100. Nagai, H.; Haga, T.; Giovannini, G.; Doi, A.; Orienti, M.; D’Ammando, F.; Kino, M.; Nakamura, M.; Asada, K.; Hada, K.; et al. Limb-brightened Jet of 3C 84 Revealed by the $43 \mathrm{GHz}$ Very-Long-Baseline-Array Observation. Astrophys. J. 2014, 785, 53. [CrossRef]

101. Giovannini, G.; Savolainen, T.; Orienti, M.; Nakamura, M.; Nagai, H.; Kino, M.; Giroletti, M.; Hada, K.; Bruni, G.; Kovalev, Y.Y.; et al. A wide and collimated radio jet in 3C 84 on the scale of a few hundred gravitational radii. Nat. Astron. 2018, 2, 472-477. [CrossRef]

102. Kino, M.; Wajima, K.; Kawakatu, N.; Nagai, H.; Orienti, M.; Giovannini, G.; Hada, K.; Niinuma, K.; Giroletti, M. Evidence of Jet-Clump Interaction: A Flip of the Radio Jet Head of 3C 84. Astrophys. J. 2018, 864, 118. [CrossRef]

103. Hiura, K.; Nagai, H.; Kino, M.; Niinuma, K.; Sorai, K.; Chida, H.; Akiyama, K.; D'Ammando, F.; Giovannini, G.; Giroletti, M.; et al. VERA monitoring of the radio jet 3C 84 in the period of 2007-2013: Detection of non-linear motion. Publ. Astron. Soc. Jpn. 2018, 70, 83. [CrossRef]

104. Hodgson, J.A.; Rani, B.; Lee, S.S.; Algaba, J.C.; Kino, M.; Trippe, S.; Park, J.H.; Zhao, G.Y.; Byun, D.Y.; Kang, S.; et al. KVN observations reveal multiple $\gamma$-ray emission regions in 3C 84? Mon. Not. R. Astron. Soc. 2018, 475, 368-378. [CrossRef]

105. Nagai, H.; Fujita, Y.; Nakamura, M.; Orienti, M.; Kino, M.; Asada, K.; Giovannini, G. Enhanced Polarized Emission from the One-parsec-scale Hotspot of 3C 84 as a Result of the Interaction with the Clumpy Ambient Medium. Astrophys. J. 2017, 849, 52. [CrossRef]

106. Wagner, A.Y.; Bicknell, G.V. Relativistic Jet Feedback in Evolving Galaxies. Astrophys. J. 2011, 728, 29. [CrossRef]

107. Fujita, Y.; Nagai, H. Discovery of a new subparsec counterjet in NGC 1275: The inclination angle and the environment. Mon. Not. R. Astron. Soc. 2017, 465, L94-L98. [CrossRef]

108. Kim, J.Y.; Krichbaum, T.P.; Marscher, A.P.; Jorstad, S.G.; Agudo, I.; Thum, C.; Hodgson, J.A.; MacDonald, N.R.; Ros, E.; Lu, R.S.; et al. Spatially resolved origin of millimeter-wave linear polarization in the nuclear region of 3C 84. Astron. Astrophys. 2019, 622, A196. [CrossRef]

109. Haga, T.; Doi, A.; Murata, Y.; Sudou, H.; Kameno, S.; Hada, K. Determination of Central Engine Position and Accretion Disk Structure in NGC 4261 by Core Shift Measurements. Astrophys. J. 2015, 807, 15. [CrossRef] 
110. Nakahara, S.; Doi, A.; Murata, Y.; Hada, K.; Nakamura, M.; Asada, K. Finding Transitions of Physical Condition in Jets from Observations over the Range of $10^{3}-10^{9}$ Schwarzschild Radii in Radio Galaxy NGC 4261. Astrophys. J. 2018, 854, 148. [CrossRef]

111. Tseng, C.Y.; Asada, K.; Nakamura, M.; Pu, H.Y.; Algaba, J.C.; Lo, W.P. Structural Transition in the NGC 6251 Jet: An Interplay with the Supermassive Black Hole and Its Host Galaxy. Astrophys. J. 2016, 833, 288. [CrossRef]

112. Baczko, A.K.; Schulz, R.; Kadler, M.; Ros, E.; Perucho, M.; Krichbaum, T.P.; Böck, M.; Bremer, M.; Grossberger, C.; Lindqvist, M.; et al. A highly magnetized twin-jet base pinpoints a supermassive black hole. Astron. Astrophys. 2016, 593, A47. [CrossRef]

113. Baczko, A.K.; Schulz, R.; Kadler, M.; Ros, E.; Perucho, M.; Fromm, C.M.; Wilms, J. Asymmetric jet production in the Active Galactic Nucleus of NGC1052. Astron. Astrophys. 2019, 623, A27. [CrossRef]

114. Nakahara, S.; Doi, A.; Murata, Y.; Nakamura, M.; Hada, K.; Asada, K.; Sawada-Satoh, S.; Kameno, S. The Two-sided Jet Structures of NGC 1052 at Scales from 300 to $4 \times 10^{7}$ Schwarzschild Radii. arXiv 2019, arXiv:1909.12510.

115. Boccardi, B.; Krichbaum, T.P.; Bach, U.; Mertens, F.; Ros, E.; Alef, W.; Zensus, J.A. The stratified two-sided jet of Cygnus A. Acceleration and collimation. Astron. Astrophys. 2016, 585, A33. [CrossRef]

116. Boccardi, B.; Krichbaum, T.P.; Bach, U.; Bremer, M.; Zensus, J.A. First 3 mm-VLBI imaging of the two-sided jet in Cygnus A. Zooming into the launching region. Astron. Astrophys. 2016, 588, L9. [CrossRef]

117. Nakahara, S.; Doi, A.; Murata, Y.; Nakamura, M.; Hada, K.; Asada, K. The Cygnus A Jet: Parabolic Streamlines up to Kiloparsec Scales. Astrophys. J. 2019, 878, 61. [CrossRef]

118. Horiuchi, S.; Meier, D.L.; Preston, R.A.; Tingay, S.J. Ten Milliparsec-Scale Structure of the Nucleus Region in Centaurus A. Publ. Astron. Soc. Jpn. 2006, 58, 211-216. [CrossRef]

119. Müller, C.; Kadler, M.; Ojha, R.; Wilms, J.; Böck, M.; Edwards, P.G.; Fromm, C.M.; Hase, H.; Horiuchi, S.; Katz, U.; et al. Dual-frequency VLBI study of Centaurus A on sub-parsec scales. The highest-resolution view of an extragalactic jet. Astron. Astrophys. 2011, 530, L11. [CrossRef]

120. Müller, C.; Kadler, M.; Ojha, R.; Perucho, M.; Großberger, C.; Ros, E.; Wilms, J.; Blanchard, J.; Böck, M.; Carpenter, B.; et al. TANAMI monitoring of Centaurus A: The complex dynamics in the inner parsec of an extragalactic jet. Astron. Astrophys. 2014, 569, A115. [CrossRef]

121. Beuchert, T.; Kadler, M.; Perucho, M.; Großberger, C.; Schulz, R.; Agudo, I.; Casadio, C.; Gómez, J.L.; Gurwell, M.; Homan, D.; et al. VLBA polarimetric monitoring of 3C 111. Astron. Astrophys. 2018, 610, A32. [CrossRef]

122. Kovalev, Y.Y.; Pushkarev, A.B.; Nokhrina, E.E.; Plavin, A.V.; Beskin, V.S.; Chernoglazov, A.; Lister, M.L.; Savolainen, T. Discovery of geometry transition in nearby AGN jets. arXiv 2019, arXiv:1907.01485.

123. Gómez, J.L.; Marscher, A.P.; Alberdi, A.; Jorstad, S.G.; Agudo, I. Monthly 43 GHz VLBA Polarimetric Monitoring of 3C 120 over 16 Epochs: Evidence for Trailing Shocks in a Relativistic Jet. Astrophys. J. 2001, 561, L161-L164. [CrossRef]

124. Casadio, C.; Gómez, J.L.; Grandi, P.; Jorstad, S.G.; Marscher, A.P.; Lister, M.L.; Kovalev, Y.Y.; Savolainen, T.; Pushkarev, A.B. The Connection between the Radio Jet and the Gamma-ray Emission in the Radio Galaxy 3C 120. Astrophys. J. 2015, 808, 162. [CrossRef]

125. Boccardi, B.; Migliori, G.; Grandi, P.; Torresi, E.; Mertens, F.; Karamanavis, V.; Angioni, R.; Vignali, C. The TeV-emitting radio galaxy 3C 264. VLBI kinematics and SED modeling. Astron. Astrophys. 2019, 627, A89. [CrossRef]

126. Tchekhovskoy, A.; Bromberg, O. Three-dimensional relativistic MHD simulations of active galactic nuclei jets: Magnetic kink instability and Fanaroff-Riley dichotomy. Mon. Not. R. Astron. Soc. 2016, 461, L46-L50. [CrossRef]

127. Rani, B. Radio Galaxies-The TeV Challenge. Galaxies 2019, 7, 23. [CrossRef]

128. Hartman, R.C.; Bertsch, D.L.; Bloom, S.D.; Chen, A.W.; Deines-Jones, P.; Esposito, J.A.; Fichtel, C.E.; Friedlander, D.P.; Hunter, S.D.; McDonald, L.M.; et al. The Third EGRET Catalog of High-Energy Gamma-Ray Sources. Astrophys. J. Suppl. 1999, 123, 79-202. [CrossRef]

129. Lister, M.L.; Homan, D.C. MOJAVE: Monitoring of Jets in Active Galactic Nuclei with VLBA Experiments. I. First-Epoch $15 \mathrm{GHz}$ Linear Polarization Images. Astron. J. 2005, 130, 1389-1417. [CrossRef]

130. Lister, M.L.; Aller, M.F.; Aller, H.D.; Hodge, M.A.; Homan, D.C.; Kovalev, Y.Y.; Pushkarev, A.B.; Savolainen, T. MOJAVE. XV. VLBA $15 \mathrm{GHz}$ Total Intensity and Polarization Maps of 437 Parsec-scale AGN Jets from 1996 to 2017. Astrophys. J. Suppl. Ser. 2018, 234, 12. [CrossRef] 
131. Kellermann, K.I.; Vermeulen, R.C.; Zensus, J.A.; Cohen, M.H. Sub-Milliarcsecond Imaging of Quasars and Active Galactic Nuclei. Astron. J. 1998, 115, 1295-1318. [CrossRef]

132. Homan, D.C.; Lister, M.L.; Kovalev, Y.Y.; Pushkarev, A.B.; Savolainen, T.; Kellermann, K.I.; Richards, J.L.; Ros, E. MOJAVE. XII. Acceleration and Collimation of Blazar Jets on Parsec Scales. Astrophys. J. 2015, 798, 134. [CrossRef]

133. Lister, M.L.; Aller, M.F.; Aller, H.D.; Homan, D.C.; Kellermann, K.I.; Kovalev, Y.Y.; Pushkarev, A.B.; Richards, J.L.; Ros, E.; Savolainen, T. MOJAVE: XIII. Parsec-scale AGN Jet Kinematics Analysis Based on 19 years of VLBA Observations at 15 GHz. Astron. J. 2016, 152, 12. [CrossRef]

134. Lister, M.L.; Homan, D.C.; Hovatta, T.; Kellermann, K.I.; Kiehlmann, S.; Kovalev, Y.Y.; Max-Moerbeck, W.; Pushkarev, A.B.; Readhead, A.C.S.; Ros, E.; et al. MOJAVE. XVII. Jet Kinematics and Parent Population Properties of Relativistically Beamed Radio-Loud Blazars. Astrophys. J. 2019, 43. [CrossRef]

135. Cohen, M.H.; Meier, D.L.; Arshakian, T.G.; Homan, D.C.; Hovatta, T.; Kovalev, Y.Y.; Lister, M.L.; Pushkarev, A.B.; Richards, J.L.; Savolainen, T. Studies of the Jet in Bl Lacertae. I. Recollimation Shock and Moving Emission Features. Astrophys. J. 2014, 787, 151. [CrossRef]

136. Pushkarev, A.B.; Kovalev, Y.Y.; Lister, M.L.; Savolainen, T. MOJAVE-XIV. Shapes and opening angles of AGN jets. Mon. Not. R. Astron. Soc. 2017, 468, 4992-5003. [CrossRef]

137. Savolainen, T.; Homan, D.C.; Hovatta, T.; Kadler, M.; Kovalev, Y.Y.; Lister, M.L.; Ros, E.; Zensus, J.A. Relativistic beaming and gamma-ray brightness of blazars. Astron. Astrophys. 2010, 512, A24. [CrossRef]

138. Jorstad, S.G.; Marscher, A.P.; Morozova, D.A.; Troitsky, I.S.; Agudo, I.; Casadio, C.; Foord, A.; Gómez, J.L.; MacDonald, N.R.; Molina, S.N.; et al. Kinematics of Parsec-scale Jets of Gamma-Ray Blazars at $43 \mathrm{GHz}$ within the VLBA-BU-BLAZAR Program. Astrophys. J. 2017, 846, 98. [CrossRef]

139. Zamaninasab, M.; Clausen-Brown, E.; Savolainen, T.; Tchekhovskoy, A. Dynamically important magnetic fields near accreting supermassive black holes. Nature 2014, 510, 126-128. [CrossRef]

140. Marscher, A.P.; Jorstad, S.G.; D'Arcangelo, F.D.; Smith, P.S.; Williams, G.G.; Larionov, V.M.; Oh, H.; Olmstead, A.R.; Aller, M.F.; Aller, H.D.; et al. The inner jet of an active galactic nucleus as revealed by a radio-to- $\gamma$-ray outburst. Nature 2008, 452, 966-969. [CrossRef]

141. Marscher, A.P.; Jorstad, S.G.; Larionov, V.M.; Aller, M.F.; Aller, H.D.; Lähteenmäki, A.; Agudo, I.; Smith, P.S.; Gurwell, M.; Hagen-Thorn, V.A.; et al. Probing the Inner Jet of the Quasar PKS 1510-089 with Multi-Waveband Monitoring During Strong Gamma-Ray Activity. Astrophys. J. 2010, 710, L126-L131. [CrossRef]

142. Agudo, I.; Jorstad, S.G.; Marscher, A.P.; Larionov, V.M.; Gómez, J.L.; Lähteenmäki, A.; Gurwell, M.; Smith, P.S.; Wiesemeyer, H.; Thum, C.; et al. Location of $\gamma$-ray Flare Emission in the Jet of the BL Lacertae Object OJ287 More than 14 pc from the Central Engine. Astrophys. J. 2011, 726, L13. [CrossRef]

143. Orienti, M.; Koyama, S.; D’Ammando, F.; Giroletti, M.; Kino, M.; Nagai, H.; Venturi, T.; Dallacasa, D.; Giovannini, G.; Angelakis, E.; et al. Radio and $\gamma$-ray follow-up of the exceptionally high-activity state of PKS 1510-089 in 2011. Mon. Not. R. Astron. Soc. 2013, 428, 2418-2429. [CrossRef]

144. Casadio, C.; Gómez, J.L.; Jorstad, S.G.; Marscher, A.P.; Larionov, V.M.; Smith, P.S.; Gurwell, M.A.; Lähteenmäki, A.; Agudo, I.; Molina, S.N.; et al. A Multi-wavelength Polarimetric Study of the Blazar CTA 102 during a Gamma-Ray Flare in 2012. Astrophys. J. 2015, 813, 51. [CrossRef]

145. Lobanov, A.P.; Gómez, J.L.; Bruni, G.; Kovalev, Y.Y.; Anderson, J.; Bach, U.; Kraus, A.; Zensus, J.A.; Lisakov, M.M.; Sokolovsky, K.V.; et al. RadioAstron space VLBI imaging of polarized radio emission in the high-redshift quasar 0642+449 at 1.6 GHz. Astron. Astrophys. 2015, 583, A100. [CrossRef]

146. Gómez, J.L.; Lobanov, A.P.; Bruni, G.; Kovalev, Y.Y.; Marscher, A.P.; Jorstad, S.G.; Mizuno, Y.; Bach, U.; Sokolovsky, K.V.; Anderson, J.M.; et al. Probing the Innermost Regions of AGN Jets and Their Magnetic Fields with RadioAstron. I. Imaging BL Lacertae at 21 Microarcsecond Resolution. Astrophys. J. 2016, 817, 96. [CrossRef]

147. Kovalev, Y.Y.; Kardashev, N.S.; Kellermann, K.I.; Lobanov, A.P.; Johnson, M.D.; Gurvits, L.I.; Voitsik, P.A.; Zensus, J.A.; Anderson, J.M.; Bach, U.; et al. RadioAstron Observations of the Quasar 3C273: A Challenge to the Brightness Temperature Limit. Astrophys. J. 2016, 820, L9. [CrossRef]

148. Bruni, G.; Gómez, J.L.; Casadio, C.; Lobanov, A.; Kovalev, Y.Y.; Sokolovsky, K.V.; Lisakov, M.M.; Bach, U.; Marscher, A.; Jorstad, S.; et al. Probing the innermost regions of AGN jets and their magnetic fields with RadioAstron. II. Observations of 3C 273 at minimum activity. Astron. Astrophys. 2017, 604, A111. [CrossRef]

149. Kravchenko, E.V.; Gómez, J.L.; Kovalev, Y.Y.; Voytsik, P.A. The jet of S5 0716+71 at $\mu$ as scales with RadioAstron. arXiv 2019, arXiv:1902.04369. 
150. Casadio, C.; Marscher, A.P.; Jorstad, S.G.; Blinov, D.A.; MacDonald, N.R.;Krichbaum, T.P.; Boccardi, B.; Traianou, E.; Gómez, J.L.; Agudo, I.; et al. The magnetic field structure in CTA 102 from high resolution mm-VLBI observations during the flaring state in 2016-2017. Astron. Astrophys. 2019, 622, A158, [CrossRef]

151. Rani, B.; Krichbaum, T.P.; Marscher, A.P.; Jorstad, S.G.; Hodgson, J.A.; Fuhrmann, L.; Zensus, J.A. Jet outflow and gamma-ray emission correlations in S5 0716+714. Astron. Astrophys. 2014, 571, L2. [CrossRef]

152. Giroletti, M.; Giovannini, G.; Cotton, W.D.; Taylor, G.B.; Pérez-Torres, M.A.; Chiaberge, M.; Edwards, P.G. The jet of Markarian 501 from millions of Schwarzschild radii down to a few hundreds. Astron. Astrophys. 2008, 488, 905-914. [CrossRef]

153. Koyama, S.; Kino, M.; Giroletti, M.; Doi, A.; Giovannini, G.; Orienti, M.; Hada, K.; Ros, E.; Niinuma, K.; Nagai, H.; et al. Discovery of off-axis jet structure of TeV blazar Mrk 501 with mm-VLBI. Astron. Astrophys. 2016, 586, A113. [CrossRef]

154. Fromm, C.M.; Perucho, M.; Ros, E.; Savolainen, T.; Zensus, J.A. On the location of the supermassive black hole in CTA 102. Astron. Astrophys. 2015, 576, A43. [CrossRef]

155. Agudo, I.; Bach, U.; Krichbaum, T.P.; Marscher, A.P.; Gonidakis, I.; Diamond, P.J.; Perucho, M.; Alef, W.; Graham, D.A.; Witzel, A.; et al. Superluminal non-ballistic jet swing in the quasar NRAO 150 revealed by mm-VLBI. Astron. Astrophys. 2007, 476, L17-L20.

156. Molina, S.N.; Agudo, I.; Gómez, J.L.; Krichbaum, T.P.; Martí-Vidal, I.; Roy, A.L. Evidence of internal rotation and a helical magnetic field in the jet of the quasar NRAO 150. Astron. Astrophys. 2014, 566, A26. [CrossRef]

157. Martí-Vidal, I.; Krichbaum, T.P.; Marscher, A.; Alef, W.; Bertarini, A.; Bach, U.; Schinzel, F.K.; Rottmann, H.; Anderson, J.M.; Zensus, J.A.; et al. On the calibration of full-polarization $86 \mathrm{GHz}$ global VLBI observations. Astron. Astrophys. 2012, 542, A107. [CrossRef]

158. Hodgson, J.A.; Krichbaum, T.P.; Marscher, A.P.; Jorstad, S.G.; Rani, B.; Marti-Vidal, I.; Bach, U.; Sanchez, S.; Bremer, M.; Lindqvist, M.; et al. Location of $\gamma$-ray emission and magnetic field strengths in OJ 287. Astron. Astrophys. 2017, 597, A80. [CrossRef]

159. Casadio, C.; Krichbaum, T.; Marscher, A.; Jorstad, S.; Gómez, J.; Agudo, I.; Bach, U.; Kim, J.Y.; Hodgson, J.; Zensus, A. 3 mm GMVA Observations of Total and Polarized Emission from Blazar and Radio Galaxy Core Regions. Galaxies 2017, 5, 67. [CrossRef]

160. Han, S.T.; Lee, J.W.; Kang, J.; Je, D.H.; Chung, M.H.; Wi, S.O.; Sasao, T.; Wylde, R. Millimeter-wave Receiver Optics for Korean VLBI Network. Int. J. Infrared Millim. Waves 2008, 29, 69-78. [CrossRef]

161. Lee, S.S.; Wajima, K.; Algaba, J.C.; Zhao, G.Y.; Hodgson, J.A.; Kim, D.W.; Park, J.; Kim, J.Y.; Miyazaki, A.; Byun, D.Y.; et al. Interferometric Monitoring of Gamma-Ray Bright AGNs. I. The Results of Single-epoch Multifrequency Observations. Astrophys. J. Suppl. Ser. 2016, 227, 8. [CrossRef]

162. Park, J.; Kam, M.; Trippe, S.; Kang, S.; Byun, D.Y.; Kim, D.W.; Algaba, J.C.; Lee, S.S.; Zhao, G.Y.; Kino, M.; et al. Revealing the Nature of Blazar Radio Cores through Multifrequency Polarization Observations with the Korean VLBI Network. Astrophys. J. 2018, 860, 112. [CrossRef]

163. Blandford, R.D.; Königl, A. Relativistic jets as compact radio sources. Astrophys. J. 1979, 232, 34-48. [CrossRef]

164. Dodson, R.; Rioja, M.J.; Molina, S.N.; Gómez, J.L. High-precision Astrometric Millimeter Very Long Baseline Interferometry Using a New Method for Multi-frequency Calibration. Astrophys. J. 2017, 834, 177. [CrossRef]

165. O'Sullivan, S.P.; Gabuzda, D.C. Three-dimensional magnetic field structure of six parsec-scale active galactic nuclei jets. Mon. Not. R. Astron. Soc. 2009, 393, 429-456. [CrossRef]

166. Sokolovsky, K.V.; Kovalev, Y.Y.; Pushkarev, A.B.; Lobanov, A.P. A VLBA survey of the core shift effect in AGN jets. I. Evidence of dominating synchrotron opacity. Astron. Astrophys. 2011, 532, A38. [CrossRef]

167. Pushkarev, A.B.; Hovatta, T.; Kovalev, Y.Y.; Lister, M.L.; Lobanov, A.P.; Savolainen, T.; Zensus, J.A. MOJAVE: Monitoring of Jets in Active galactic nuclei with VLBA Experiments. IX. Nuclear opacity. Astron. Astrophys. 2012, 545, A113. [CrossRef]

168. Lobanov, A.P. Ultracompact jets in active galactic nuclei. Astron. Astrophys. 1998, 330, 79-89.

169. Hirotani, K. Kinetic Luminosity and Composition of Active Galactic Nuclei Jets. Astrophys. J. 2005, 619, 73-85. [CrossRef]

170. Algaba, J.C.; Nakamura, M.; Asada, K.; Lee, S.S. Resolving the Geometry of the Innermost Relativistic Jets in Active Galactic Nuclei. Astrophys. J. 2017, 834, 65. [CrossRef]

171. Zavala, R.T.; Taylor, G.B. Faraday Rotation Measures in the Parsec-Scale Jets of the Radio Galaxies M87, 3C 111, and 3C 120. Astrophys. J. 2002, 566, L9-L12. [CrossRef] 
172. Blandford, R.D. Astrophysical Jets; Burgarella D., Livio M., O’Dea C., Eds.; Cambridge University Press: Cambridge, UK, 1993; p. 26.

173. Broderick, A.E.; McKinney, J.C. Parsec-scale Faraday Rotation Measures from General Relativistic Magnetohydrodynamic Simulations of Active Galactic Nucleus Jets. Astrophys. J. 2010, 725, 750-773. [CrossRef]

174. Asada, K.; Inoue, M.; Uchida, Y.; Kameno, S.; Fujisawa, K.; Iguchi, S.; Mutoh, M. A Helical Magnetic Field in the Jet of 3C 273. Publ. Astron. Soc. Jpn. 2002, 54, L39-L43. [CrossRef]

175. Gabuzda, D.C.; Murray, É.; Cronin, P. Helical magnetic fields associated with the relativistic jets of four BL Lac objects. Mon. Not. R. Astron. Soc. 2004, 351, L89-L93. [CrossRef]

176. Asada, K.; Inoue, M.; Nakamura, M.; Kameno, S.; Nagai, H. Multifrequency Polarimetry of the NRAO 140 Jet: Possible Detection of a Helical Magnetic Field and Constraints on Its Pitch Angle. Astrophys. J. 2008, 682, 798-802. [CrossRef]

177. Mahmud, M.; Gabuzda, D.C.; Bezrukovs, V. Surprising evolution of the parsec-scale Faraday Rotation gradients in the jet of the BL Lac object B1803+784. Mon. Not. R. Astron. Soc. 2009, 400, 2-12. [CrossRef]

178. Croke, S.M.; O'Sullivan, S.P.; Gabuzda, D.C. The parsec-scale distributions of intensity, linear polarization and Faraday rotation in the core and jet of Mrk501 at 8.4-1.6 GHz. Mon. Not. R. Astron. Soc. 2010, 402, 259-270. [CrossRef]

179. Hovatta, T.; Lister, M.L.; Aller, M.F.; Aller, H.D.; Homan, D.C.; Kovalev, Y.Y.; Pushkarev, A.B.; Savolainen, T. MOJAVE: Monitoring of Jets in Active Galactic Nuclei with VLBA Experiments. VIII. Faraday Rotation in Parsec-scale AGN Jets. Astron. J. 2012, 144, 105. [CrossRef]

180. Zamaninasab, M.; Savolainen, T.; Clausen-Brown, E.; Hovatta, T.; Lister, M.L.; Krichbaum, T.P.; Kovalev, Y.Y.; Pushkarev, A.B. Evidence for a large-scale helical magnetic field in the quasar 3C 454.3. Mon. Not. R. Astron. Soc. 2013, 436, 3341-3356. [CrossRef]

181. Gabuzda, D.C.; Knuettel, S.; Reardon, B. Transverse Faraday-rotation gradients across the jets of 15 active galactic nuclei. Mon. Not. R. Astron. Soc. 2015, 450, 2441-2450. [CrossRef]

182. Gabuzda, D.C.; Roche, N.; Kirwan, A.; Knuettel, S.; Nagle, M.; Houston, C. Parsec scale Faraday-rotation structure across the jets of nine active galactic nuclei. Mon. Not. R. Astron. Soc. 2017, 472, 1792-1801. [CrossRef]

183. Martí-Vidal, I.; Muller, S.; Vlemmings, W.; Horellou, C.; Aalto, S. A strong magnetic field in the jet base of a supermassive black hole. Science 2015, 348, 311-314. [CrossRef] [PubMed]

184. Hovatta, T.; O'Sullivan, S.; Martí-Vidal, I.; Savolainen, T.; Tchekhovskoy, A. Magnetic field at a jet base: Extreme Faraday rotation in 3C 273 revealed by ALMA. Astron. Astrophys. 2019, 623, A111. [CrossRef]

185. Abdo, A.A.; Ackermann, M.; Ajello, M.; Baldini, L.; Ballet, J.; Barbiellini, G.; Bastieri, D.; Bechtol, K.; Bellazzini, R.; Berenji, B.; et al. Radio-Loud Narrow-Line Seyfert 1 as a New Class of Gamma-Ray Active Galactic Nuclei. Astrophys. J. 2009, 707, L142-L147. [CrossRef]

186. Osterbrock, D.E.; Pogge, R.W. The spectra of narrow-line Seyfert 1 galaxies. Astrophys. J. 1985, 297, $166-176$. [CrossRef]

187. Boller, T.; Brandt, W.N.; Fink, H. Soft X-ray properties of narrow-line Seyfert 1 galaxies. Astron. Astrophys. 1996, 305, 53.

188. Komossa, S.; Voges, W.; Xu, D.; Mathur, S.; Adorf, H.M.; Lemson, G.; Duschl, W.J.; Grupe, D. Radio-loud Narrow-Line Type 1 Quasars. Astron. J. 2006, 132, 531-545. [CrossRef]

189. Paliya, V.S.; Ajello, M.; Rakshit, S.; Mand al, A.K.; Stalin, C.S.; Kaur, A.; Hartmann, D. Gamma-Ray-emitting Narrow-line Seyfert 1 Galaxies in the Sloan Digital Sky Survey. Astrophys. J. 2018, 853, L2. [CrossRef]

190. D'Ammando, F. Relativistic Jets in Gamma-Ray-Emitting Narrow-Line Seyfert 1 Galaxies. Galaxies 2019, 7, 87. [CrossRef]

191. Doi, A.; Nagai, H.; Asada, K.; Kameno, S.; Wajima, K.; Inoue, M. VLBI Observations of the Most Radio-Loud, Narrow-Line Quasar SDSS J094857.3+002225. Publ. Astron. Soc. Jpn. 2006, 58, 829-834. [CrossRef]

192. Doi, A.; Fujisawa, K.; Inoue, M.; Wajima, K.; Nagai, H.; Harada, K.; Suematsu, K.; Habe, A.; Honma, M.; Kawaguchi, N.; et al. Japanese VLBI Network Observations of Radio-Loud Narrow-Line Seyfert 1 Galaxies. Publ. Astron. Soc. Jpn. 2007, 59, 703-709. [CrossRef]

193. Doi, A.; Asada, K.; Nagai, H. Very Long Baseline Array Imaging of Parsec-scale Jet Structures in Radio-loud Narrow-line Seyfert 1 Galaxies. Astrophys. J. 2011, 738, 126. [CrossRef] 
194. Giroletti, M.; Paragi, Z.; Bignall, H.; Doi, A.; Foschini, L.; Gabányi, K.É.; Reynolds, C.; Blanchard, J.; Campbell, R.M.; Colomer, F.; et al. Global e-VLBI observations of the gamma-ray narrow line Seyfert 1 PMN J0948+0022. Astron. Astrophys. 2011, 528, L11. [CrossRef]

195. Foschini, L.; Ghisellini, G.; Kovalev, Y.Y.; Lister, M.L.; D’Ammando, F.; Thompson, D.J.; Tramacere, A.; Angelakis, E.; Donato, D.; Falcone, A.; et al. The first gamma-ray outburst of a narrow-line Seyfert 1 galaxy: The case of PMN J0948+0022 in 2010 July. Mon. Not. R. Astron. Soc. 2011, 413, 1671-1677. [CrossRef]

196. Orienti, M.; D'Ammando, F.; Giroletti, M. High resolution radio observations of gamma-ray emitting Narrow-Line Seyfert 1s. arXiv 2012, arXiv:1205.0402.

197. Doi, A.; Asada, K.; Fujisawa, K.; Nagai, H.; Hagiwara, Y.; Wajima, K.; Inoue, M. Very Long Baseline Array Imaging of Parsec-scale Radio Emissions in Nearby Radio-quiet Narrow-line Seyfert 1 Galaxies. Astrophys. J. 2013, 765, 69. [CrossRef]

198. D'Ammando, F.; Orienti, M.; Finke, J.; Raiteri, C.M.; Angelakis, E.; Fuhrmann, L.; Giroletti, M.; Hovatta, T.; Karamanavis, V.; Max-Moerbeck, W.; et al. Multifrequency studies of the narrow-line Seyfert 1 galaxy SBS 0846+513. Mon. Not. R. Astron. Soc. 2013, 436, 191-201. [CrossRef]

199. Wajima, K.; Fujisawa, K.; Hayashida, M.; Isobe, N.; Ishida, T.; Yonekura, Y. Short-term Radio Variability and Parsec-scale Structure in a Gamma-Ray Narrow-line Seyfert 1 Galaxy 1H 0323+342. Astrophys. J. 2014, 781, 75. [CrossRef]

200. D'Ammando, F.; Orienti, M.; Tavecchio, F.; Ghisellini, G.; Torresi, E.; Giroletti, M.; Raiteri, C.M.; Grandi, P.; Aller, M.; Aller, H.; et al. Unveiling the nature of the $\gamma$-ray emitting active galactic nucleus PKS 0521-36. Mon. Not. R. Astron. Soc. 2015, 450, 3975-3990. [CrossRef]

201. Orienti, M.; D’Ammando, F.; Larsson, J.; Finke, J.; Giroletti, M.; Dallacasa, D.; Isacsson, T.; Stoby Hoglund, J. Investigating powerful jets in radio-loud narrow-line Seyfert 1s. Mon. Not. R. Astron. Soc. 2015, 453, 4037-4050. [CrossRef]

202. Gu, M.; Chen, Y.; Komossa, S.; Yuan, W.; Shen, Z.; Wajima, K.; Zhou, H.; Zensus, J.A. The Radio Properties of Radio-loud Narrow-line Seyfert 1 Galaxies on Parsec Scales. Astrophys. J. Suppl. 2015, 221, 3. [CrossRef]

203. Richards, J.L.; Lister, M.L.; Savolainen, T.; Homan, D.C.; Kadler, M.; Hovatta, T.; Readhead, A.C.S.; Arshakian, T.G.; Chavushyan, V. The parsec-scale structure, kinematics, and polarization of radio-loud narrow-line Seyfert 1 galaxies. In IAU Symposium; Extragalactic Jets from Every Angle; Massaro, F., Cheung, C.C., Lopez, E., Siemiginowska, A., Eds.; Cambridge University Press: Cambridge, UK, 2015; Volume 313, pp. 139-142. [CrossRef]

204. Doi, A.; Oyama, T.; Kono, Y.; Yamauchi, A.; Suzuki, S.; Matsumoto, N.; Tazaki, F. A radio detection survey of narrow-line Seyfert 1 galaxies using very long baseline interferometry at $22 \mathrm{GHz}$. Publ. Astron. Soc. Jpn. 2016, 68, 73. [CrossRef]

205. Angelakis, E.; Fuhrmann, L.; Marchili, N.; Foschini, L.; Myserlis, I.; Karamanavis, V.; Komossa, S.; Blinov, D.; Krichbaum, T.P.; Sievers, A.; et al. Radio jet emission from GeV-emitting narrow-line Seyfert 1 galaxies. Astron. Astrophys. 2015, 575, A55. [CrossRef]

206. Lähteenmäki, A.; Järvelä, E.; Hovatta, T.; Tornikoski, M.; Harrison, D.L.; López-Caniego, M.; Max-Moerbeck, W.; Mingaliev, M.; Pearson, T.J.; Ramakrishnan, V.; et al. 37 GHz observations of narrow-line Seyfert 1 galaxies. Astron. Astrophys. 2017, 603, A100. [CrossRef]

207. Doi, A.; Nagira, H.; Kawakatu, N.; Kino, M.; Nagai, H.; Asada, K. Kiloparsec-scale Radio Structures in Narrow-line Seyfert 1 Galaxies. Astrophys. J. 2012, 760, 41. [CrossRef]

208. Richards, J.L.; Lister, M.L. Kiloparsec-Scale Jets in Three Radio-Loud Narrow-Line Seyfert 1 Galaxies. Astrophys. J. 2015, 800, L8. [CrossRef]

209. Berton, M.; Congiu, E.; Järvelä, E.; Antonucci, R.; Kharb, P.; Lister, M.L.; Tarchi, A.; Caccianiga, A.; Chen, S.; Foschini, L.; et al. Radio-emitting narrow-line Seyfert 1 galaxies in the JVLA perspective. Astron. Astrophys. 2018, 614, A87. [CrossRef]

210. Foschini, L.; Ciroi, S.; Berton, M.; Vercellone, S.; Romano, P.; Braito, V. Mapping the Narrow-Line Seyfert 1 Galaxy 1H 0323342+. Universe 2019, 5, 199. [CrossRef]

211. Zhou, H.; Wang, T.; Yuan, W.; Shan, H.; Komossa, S.; Lu, H.; Liu, Y.; Xu, D.; Bai, J.M.; Jiang, D.R. A Narrow-Line Seyfert 1-Blazar Composite Nucleus in 2MASX J0324+3410. Astrophys. J. 2007, 658, L13-L16. [CrossRef] 
212. Wang, F.; Du, P.; Hu, C.; Bai, J.M.; Wang, C.J.; Yi, W.M.; Wang, J.G.; Zhang, J.J.; Xin, Y.X.; Lun, B.L.; et al. Reverberation Mapping of the Gamma-Ray Loud Narrow-line Seyfert 1 Galaxy 1H 0323+342. Astrophys. J. 2016, 824, 149. [CrossRef]

213. Yao, S.; Yuan, W.; Komossa, S.; Grupe, D.; Fuhrmann, L.; Liu, B. The $\gamma$-Ray Detected Narrow-line Seyfert 1 Galaxy 1H 0323+342: Swift Monitoring and Suzaku Spectroscopy. Astron. J. 2015, 150, 23. [CrossRef]

214. Landt, H.; Ward, M.J.; Baloković, M.; Kynoch, D.; Storchi-Bergmann, T.; Boisson, C.; Done, C.; Schimoia, J.; Stern, D. On the black hole mass of the $\gamma$-ray emitting narrow-line Seyfert 1 galaxy $1 \mathrm{H} 0323+342$. Mon. Not. R. Astron. Soc. 2017, 464, 2565-2576. [CrossRef]

215. León Tavares, J.; Kotilainen, J.; Chavushyan, V.; Añorve, C.; Puerari, I.; Cruz-González, I.; Patiño-Alvarez, V.; Antón, S.; Carramiñana, A.; Carrasco, L.; et al. The Host Galaxy of the Gamma-Ray Narrow-line Seyfert 1 Galaxy 1H 0323+342. Astrophys. J. 2014, 795, 58. [CrossRef]

216. Doi, A.; Hada, K.; Kino, M.; Wajima, K.; Nakahara, S. A Recollimation Shock in a Stationary Jet Feature with Limb-brightening in the Gamma-Ray-emitting Narrow-line Seyfert 1 Galaxy 1H 0323+342. Astrophys. J. 2018, 857, L6. [CrossRef]

217. Fuhrmann, L.; Karamanavis, V.; Komossa, S.; Angelakis, E.; Krichbaum, T.P.; Schulz, R.; Kreikenbohm, A.; Kadler, M.; Myserlis, I.; Ros, E.; et al. Inner jet kinematics and the viewing angle towards the $\gamma$-ray narrow-line Seyfert 1 galaxy $1 \mathrm{H} 0323+342$. Res. Astron. Astrophys. 2016, 16, 176, [CrossRef]

218. Hada, K.; Doi, A.; Wajima, K.; D’Ammand o, F.; Orienti, M.; Giroletti, M.; Giovannini, G.; Nakamura, M.; Asada, K. Collimation, Acceleration, and Recollimation Shock in the Jet of Gamma-Ray Emitting Radio-loud Narrow-line Seyfert 1 Galaxy 1H0323+342. Astrophys. J. 2018, 860, 141. [CrossRef]

219. D'Ammando, F.; Orienti, M.; Finke, J.; Larsson, J.; Giroletti, M.; Raiteri, C. A Panchromatic View of Relativistic Jets in Narrow-Line Seyfert 1 Galaxies. Galaxies 2016, 4, 11. [CrossRef]

220. Paliya, V.S. Gamma-ray emitting narrow-line Seyfert 1 galaxies: Past, present, and future. J. Astrophys. Astron. 2019, 40, 39. [CrossRef]

221. Ohsuga, K.; Mineshige, S. Global Structure of Three Distinct Accretion Flows and Outflows around Black Holes from Two-dimensional Radiation-magnetohydrodynamic Simulations. Astrophys. J. 2011, 736, 2. [CrossRef]

222. Berton, M.; Caccianiga, A.; Foschini, L.; Peterson, B.M.; Mathur, S.; Terreran, G.; Ciroi, S.; Congiu, E.; Cracco, V.; Frezzato, M.; et al. Compact steep-spectrum sources as the parent population of flat-spectrum radio-loud narrow-line Seyfert 1 galaxies. Astron. Astrophys. 2016, 591, A98. [CrossRef]

223. Foschini, L. What we talk about when we talk about blazars? Front. Astron. Space Sci. 2017, 4, 6, [CrossRef]

224. Lico, R.; Giroletti, M.; Orienti, M.; Costamante, L.; Pavlidou, V.; D’Ammando, F.; Tavecchio, F. Exploring the connection between radio and GeV-TeV $\gamma$-ray emission in the 1FHL and 2FHL AGN samples. Astron. Astrophys. 2017, 606, A138. [CrossRef]

225. Ghisellini, G.; Tavecchio, F.; Chiaberge, M. Structured jets in TeV BL Lac objects and radiogalaxies. Implications for the observed properties. Astron. Astrophys. 2005, 432, 401-410. [CrossRef]

226. Nagar, N.M.; Falcke, H.; Wilson, A.S. Radio sources in low-luminosity active galactic nuclei. IV. Radio luminosity function, importance of jet power, and radio properties of the complete Palomar sample. Astron. Astrophys. 2005, 435, 521-543. [CrossRef]

227. Ho, L.C. Nuclear activity in nearby galaxies. Annu. Rev. Astron. Astrophys. 2008, 46, 475-539. [CrossRef]

228. Issaoun, S.; Johnson, M.D.; Blackburn, L.; Brinkerink, C.D.; Mościbrodzka, M.; Chael, A.; Goddi, C.; Martí-Vidal, I.; Wagner, J.; Doeleman, S.S.; et al. The Size, Shape, and Scattering of Sagittarius A* at 86 GHz: First VLBI with ALMA. Astrophys. J. 2019, 871, 30. [CrossRef]

229. Jiang, W.; Shen, Z.; Jiang, D.; Martí-Vidal, I.; Kawaguchi, N. VLBI Imaging of M81* at $\lambda=3.4 \mathrm{~mm}$ with Source-frequency Phase-referencing. Astrophys. J. 2018, 853, L14. [CrossRef]

230. Ly, C.; Walker, R.C.; Wrobel, J.M. An Attempt to Probe the Radio Jet Collimation Regions in NGC 4278, NGC 4374 (M84), and NGC 6166. Astron. J. 2004, 127, 119-124. [CrossRef]

231. Hada, K.; Doi, A.; Nagai, H.; Inoue, M.; Honma, M.; Giroletti, M.; Giovannini, G. Evidence for a Nuclear Radio Jet and its Structure down to lsim100 Schwarzschild Radii in the Center of the Sombrero Galaxy (M 104, NGC 4594). Astrophys. J. 2013, 779, 6. [CrossRef]

232. Kormendy, J.; Ho, L.C. Coevolution (Or Not) of Supermassive Black Holes and Host Galaxies. Annu. Rev. Astron. Astrophys. 2013, 51, 511-653. [CrossRef] 
233. IceCube Collaboration; Aartsen, M.G.; Ackermann, M.; Adams, J.; Aguilar, J.A.; Ahlers, M.; Ahrens, M.; Al Samarai, I.; Altmann, D.; Andeen, K.; et al. Multimessenger observations of a flaring blazar coincident with high-energy neutrino IceCube-170922A. Science 2018, 361, eaat1378. [CrossRef]

234. Kun, E.; Biermann, P.L.; Gergely, L.Á. Very long baseline interferometry radio structure and radio brightening of the high-energy neutrino emitting blazar TXS 0506+056. Mon. Not. R. Astron. Soc. 2019, 483, L42-L46. [CrossRef]

(C) 2019 by the author. Licensee MDPI, Basel, Switzerland. This article is an open access article distributed under the terms and conditions of the Creative Commons Attribution (CC BY) license (http://creativecommons.org/licenses/by/4.0/). 